\title{
Pattern selection in a horizontally vibrated container
}

\author{
J Porter, I Tinao, A Laverón-Simavilla and C A Lopez
}

\begin{abstract}
We present results of an experimental and numerical investigation of the patterns realized by surface waves within an open rectangular container subjected to horizontal vibrations at frequencies of $40-100 \mathrm{~Hz}$. The first instability exhibited by the primary harmonic wave field is subharmonic, and may be identified with the cross-wave instability often seen in wave tank experiments. We show that, contrary to common theoretical and experimental assumptions, and despite their name, these subharmonic waves are not oriented crosswise, but at an intermediate angle with respect to the axis of vibration. Hence, the pattern selection problem for horizontally forced Faraday waves is more complex than has previously been assumed. We establish the robustness of this obliquely oriented surface wave pattern by varying the forcing frequency and amplitude, the fluid viscosity, the fluid depth, and the boundary conditions. Previous work on cross-waves is reviewed and discussed in relation to the current results. Finally, numerical simulations using a reduced model with an appropriate forcing term are used to support the generality of the experimental observations.
\end{abstract}

(Some figures may appear in color only in the online journal)

\section{Introduction}

The question of how a mass of fluid responds to external forcing is of fundamental interest in fluid dynamics. Periodic forcing, in particular, appears throughout nature and engineering applications, where it can greatly affect fluid behavior. Vertical forcing can delay

${ }^{1}$ Present Adtress: Intel Platform Technologies Lab, Guadalajata Design Center, Periférico sur 7980 4-E. Tlaquepaque. Jalisco 45600. México. 
convection (Gresho and Sani 1970), suppress the Rayleigh-Taylor instability (Wolf 1969, Wolf 1970), induce mean flows (Vega et al 2001), and affect heat or mass transfer. Horizontal forcing is associated with sloshing and other types of resonant and near-resonant mode dynamics at low frequencies (Miles 1984, Funakoshi and Inoue 1988, Feng 1997), with wavemaker experiments and cross-waves (see Miles and Henderson 1990 and references therein) at intermediate frequencies, and with frozen waves (Wolf 1969, Talib et al 2007) on the interface between two immiscible fluids of comparable densities. Interesting surface wave patterns have been observed in tubes of liquid-gas $\mathrm{CO}_{2}$ mixtures using horizontally forcing of intermediate to high frequency (González-Viñas and Salán 1994, Wunenburger et al 1999).

The surface or interfacial dynamics of periodically forced fluid systems can be divided according to system size. In small-aspect-ratio systems, where the wavelength of excited modes is comparable to the system size, behavior is typically determined by a small number of relevant modes, and is strongly influenced by geometry (container shape, for example). If, on the other hand, the interface or free surface is large compared to the wavelength of excited modes, distinct patterns may compete with one another to produce new kinds of complex dynamics. This richness is readily observed in the popular Faraday system (an open container of fluid vibrated vertically), which has proved a nearly inexhaustible source of intriguing patterns (Edwards and Fauve 1994, Kudrolli et al 1998, Arbell and Fineberg 2002) long after Faraday's seminal experiments (Faraday 1831). Although Faraday himself investigated both vertical and horizontal vibrations, subsequent attention has clearly favored the vertical case, now regarded as a classic example of parametric instability (Benjamin and Ursell 1954). In its analysis, it is standard to assume a perfectly flat horizontal surface undergoing perfectly vertical vibrations. The forcing is uniform and, in a comoving frame, reduces to a modulation of the gravitational force. Due to planar Euclidean symmetry (in the infinitely extended limit) the motionless flat surface solution persists. If, on the other hand, the undisturbed surface is not perfectly flat, either due to capillary forces along the walls of the container or the influence of surface tension, which dominates at small scales and under microgravity conditions, then there is no Euclidean symmetry and the equilibrium surface configuration is immediately destroyed by the forcing. The same applies to a perfectly flat (and bounded) surface if the forcing is not entirely perpendicular to it. From a general perspective, it can thus be argued that the response to horizontal excitation, which is less dependent on symmetry, is more relevant to the question of how a mass of fluid responds to generic periodic forcing.

Among the diverse phenomena found in horizontally forced systems, cross-waves hold particular interest because they arise from a parametric instability (Garrett 1970) at the wavemaker (boundary) and are thus localized counterparts of vertically forced Faraday waves. Cross-waves were described by Faraday (1831) as a series of apparently permanent ridges projecting outward 'like the teeth of a coarse comb' from a partially submerged vibrating plate. They are commonly observed in wavemaker experiments (Barnard and Pritchard 1972), and can exhibit complex behavior including chaotic dynamics and slow modulations resembling solitary waves (Underhill et al 1991).

Despite a long history of attention (Faraday 1831, Schuler 1933, Lin and Howard 1960, Garrett 1970, Barnard and Pritchard 1972, Jones 1984, Lichter and Underhill 1987, Miles 1988, Shemer and Lichter 1990, Underhill et al 1991), there are few cross-wave experiments comparable to the many pattern formation experiments in the vertically forced case (i.e. at relatively high frequencies in large-aspect-ratio containers). Motivated by this, and by recent theoretical work (Varas and Vega 2007) identifying subharmonic instability in horizontally vibrated containers of one extended dimension (where no 'cross-waves' are possible), we investigate cross-wave type patterns in a horizontally vibrated square container 
whose width is larger (by a factor of ten or more) than the wavelength of the surface waves. We find that the subharmonic waves consistently avoid a crosswise orientation, instead forming patterns that are characterized by an intermediate orientation, usually between $50^{\circ}$ and $80^{\circ}$, never $90^{\circ}$. The prevalence of these oblique patterns across a wide range of experimental parameters indicates that the cross-wave instability belongs to a more general class of subharmonic instability that can generate a variety of patterns.

The paper is organized as follows. The experimental set-up is described in section 2 , followed by experimental results in section 3 where the influence of boundary conditions, fluid depth and viscosity are examined in turn. Previous theoretical and experimental work is discussed in section 4 . Because the patterns we find experimentally violate the assumptions of the existing theoretical models, we introduce an alternative model in section 5, where numerical simulations are used to provide additional insight. Conclusions are presented in section 6 .

\section{Experimental setup}

The experiments are performed in a series of transparent square acrylic containers that are $9 \mathrm{~cm}$ across inside and either $5 \mathrm{~cm}$ or $2 \mathrm{~cm}$ deep. A sharp edge (step) at the top is used to pin the contact line when desired, and is coated with an anti-wetting agent (3M Novec EGC1700). The working container is mounted on a specially constructed dual rail system and is free to slide horizontally (hereafter referred to as the streamwise or $x$-direction) in response to a VTS-150 electromagnetic shaker. The frequency is accurate to within $10^{-2} \mathrm{~Hz}$. The motion is monitored with an accelerometer (ADXL321) mounted on the wall of the container. An arrangement of lenses and mirrors provides illumination through the transparent bottom of the container. A high speed camera (Redlake MotionPro X3) records images of $700 \times 700$ pixels (spatial resolution of $0.129 \mathrm{~mm}$ ) and we take 15 snapshots over each forcing period ( 30 images for each period of the subharmonic response). These images capture the geometric features of the surface wave pattern and provide a quantitative measure of the surface deformation in the limit of small surface wave amplitude. In that limit, the relative variation in light intensity $I$ at the detector is proportional to the curvature of the surface (Settles 2001):

$$
\frac{I-I_{0}}{I_{0}}=-\beta \frac{n_{1}}{n_{2}} \nabla^{2} h(x, y)
$$

where $h(x, y)$ is the surface height, $I_{0}$ is the background illumination, $\beta$ is a geometric factor and $n_{1}\left(n_{2}\right)$ is the index of refraction of the fluid (air). Thus, local peaks in surface height appear brighter, while valleys appear darker. Equation (1) describes measurements taken sufficiently close to threshold, but will be in error if the amplitude of oscillations is high enough to cause nonlinearities in the image. Such nonlinearity is present near the endwalls, where the fluid motion is most vigorous, in most of the measurements reported below, meaning that quantities extracted from these images can only characterize the growth of the surface waves in an approximate sense.

To establish the generic nature of the observed pattern, we performed a range of measurements using DC200 silicone oil and water, using forcing frequencies between 40 and $100 \mathrm{~Hz}$. The silicone oils had kinematic viscosities of 5,10 and $50 \mathrm{cSt}$. The densities of the DC200 oils at $25^{\circ} \mathrm{C}$ are $0.913,0.933$ and $0.963 \mathrm{~g} \mathrm{~cm}^{-3}$, respectively, while typical surface tension values at that temperature are $19.7,20.1$ and $20.8 \mathrm{dyne} \mathrm{cm}^{-1}$. The frequency range of the applied forcing can be extended somewhat, but difficulties soon arise. At higher frequencies the subharmonic patterns become extremely localized near the endwalls, more so for higher viscosity fluids, making it hard to measure oscillations in the streamwise direction. 


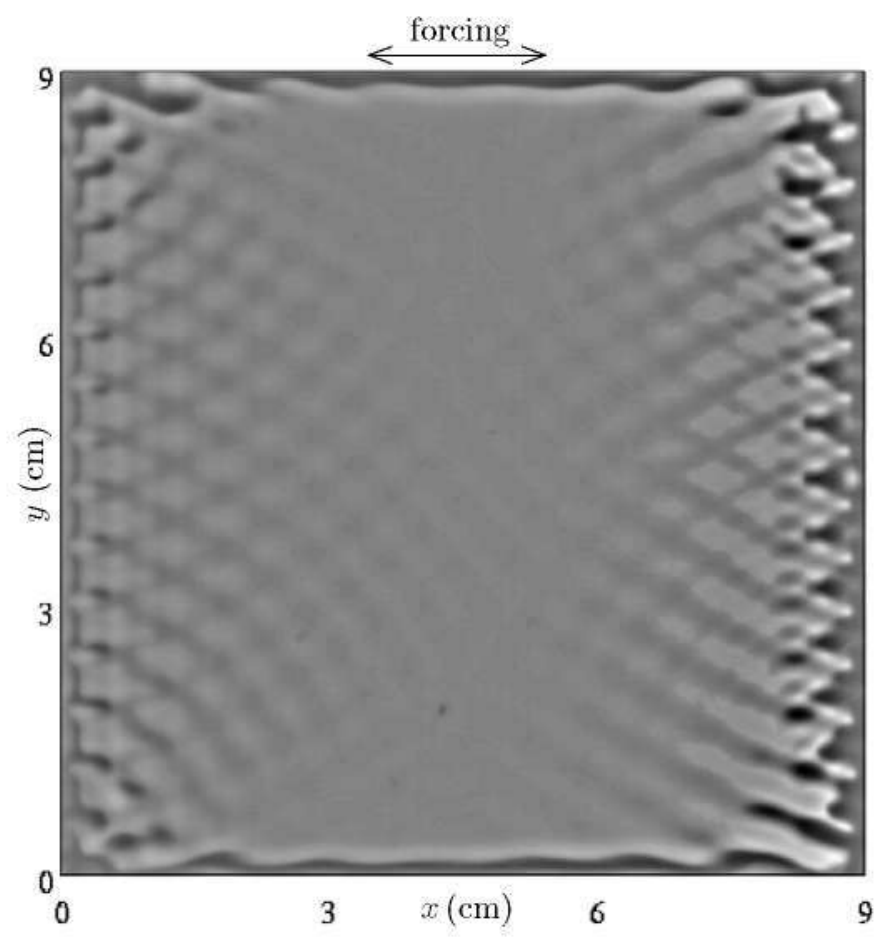

Figure 1. Snapshot of the surface wave pattern just after the subharmonic instability in a (slightly underfilled) $5 \mathrm{~cm}$ deep container of $5 \mathrm{cSt}$ oil vibrated in the $x$-direction at $70 \mathrm{~Hz}$.

At lower frequencies, particularly with lower viscosity fluids, the patterns extend throughout the container and are subject to low frequency temporal and spatial modulations, presumably due to the interaction of the wavetrains emanating from opposing sides, and this makes measurement of pattern orientation difficult.

\section{Experimental results}

Here we present our main experimental results documenting the average geometric characteristics (i.e. orientation of selected waves) of the subharmonic pattern, and the effect of varying forcing frequency and amplitude, boundary conditions, fluid depth, and fluid viscosity.

For all fluids tested, in both the 2 and $5 \mathrm{~cm}$ container, using both pinned and wetted boundary conditions, we find that the first instability suffered by the initial synchronous waves is to a subharmonic cross-wave type pattern. An example of the pattern appearing after this instability is shown in figure 1 . Note that, while the ridges immediately adjacent to the endwalls (where wave amplitude is highest) may appear to be oriented perpendicularly, there is a more complex underlying pattern that is rhomboidal, or lozenge-shaped, and shows significant variation in the $x$-direction, in contrast to the traditional picture of cross-waves. This variation is highlighted in figure 2 , which shows the profile of the subharmonic waves in both $x$ and $y$.

A Fourier transform confirms that the subharmonic waves are not oriented crosswise to the axis of vibration but, in the case of $50 \mathrm{~Hz}$ excitation shown in figure 3 , at an angle of about $70^{\circ}$. It is difficult to measure this angle precisely because of the rapid spatial decay of 


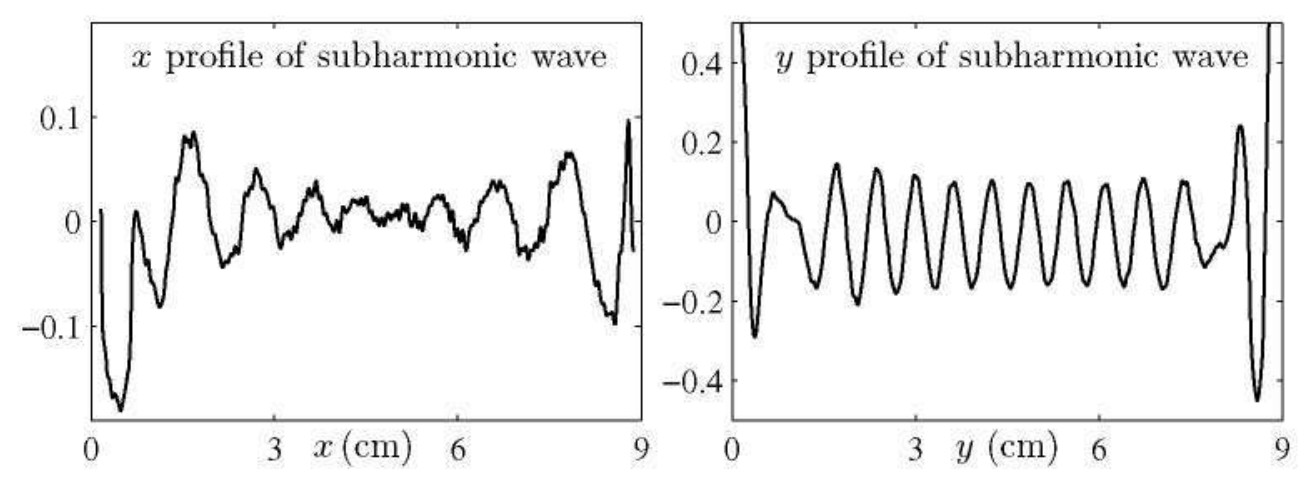

Figure 2. Profile of the instantaneous subharmonic pattern of figure 1 in $x$ (through a midplane of the container) and $y(1.2 \mathrm{~cm}$ from the left boundary), obtained after filtering to remove the harmonic waves. The images are unreliable near the boundaries due to shadows and reflections off the wall of the container.
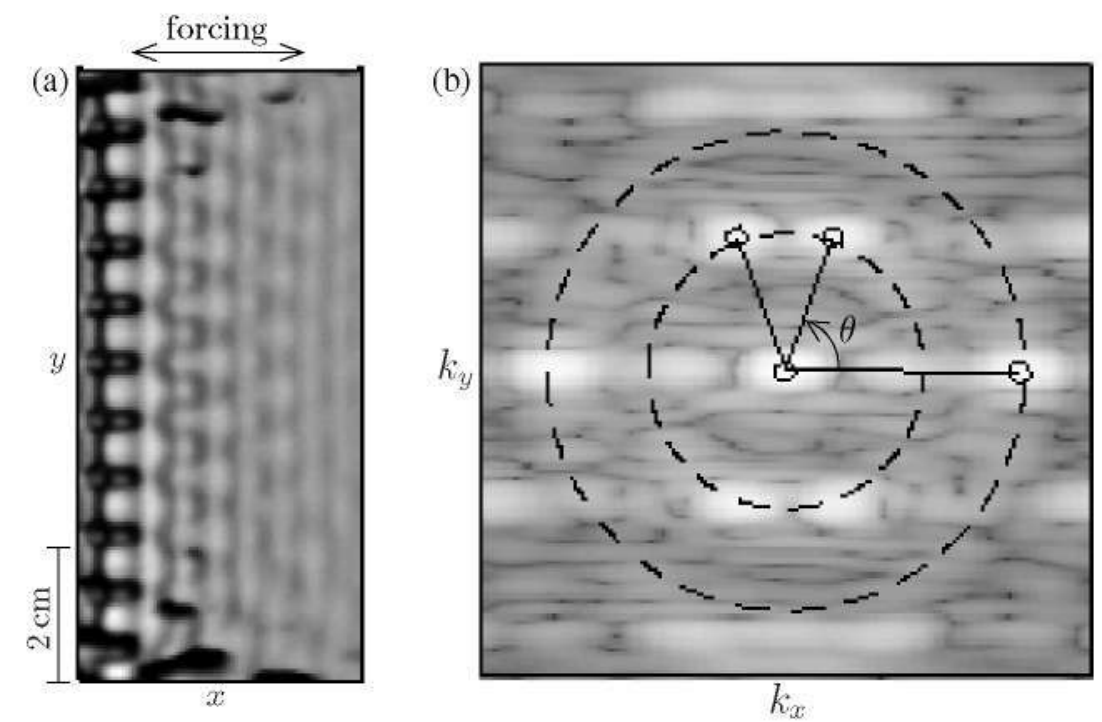

Figure 3. Instantaneous (a) surface wave pattern and (b) Fourier transform for a $5 \mathrm{~cm}$ deep layer (pinned boundary) of $5 \mathrm{cSt}$ oil vibrated at $50 \mathrm{~Hz}$. The dashed inner (outer) circle marks the wavenumber $k \simeq 9.0(k \simeq 15.7)$ predicted by the inviscid dispersion relation (see (12)) for subharmonic (harmonic) gravity-capillary waves.

the pattern —only one or two oscillations in the streamwise direction (see figure 2) are visible in many cases. Furthermore, it is unlikely that these patterns can be fully characterized by a single angle. There is sometimes evidence of multiple subharmonic peaks in the transform, and it is likely that the (local) streamwise wavenumber $k_{x}$ varies with $x$. A final difficulty is that the transform (and, specifically, the position of the subharmonic peak) varies periodically in time as the phase of the pattern within the spatial decay envelope varies. The angle given in the remainder of this section is a time average (over two forcing periods, or 30 images) of the angle obtained from the instantaneous Fourier spectrum. We note that this spectrum is obtained from an interior region that excludes a small strip (several millimeters wide or so, depending on the experiment) near the endwalls and along the lateral boundaries. This 
eliminates the highly nonlinear parts of the images that are compromised by reflections from the walls of the container. Additionally, we use subpixel interpolation (Fisher and Naidu 1996) to more accurately locate the peaks.

\subsection{Effect of boundary conditions}

The boundary conditions, especially at the endwalls, are an important factor in selecting the surface wave pattern, as demonstrated below. This is not surprising since the parametric forcing mechanism is generated by the motion of the boundary and its effect is concentrated there. Unfortunately, it is not easy to control the boundary conditions with precision because the motion of the fluid is most vigorous there (i.e. the amplitude is not small). It is particularly difficult to maintain constant boundary conditions with silicone oil, which tends to wet portions of the boundary to varying degrees as the experiment progresses. This wetting can be sudden (and visible by eye) as one or more wave crests overcome resistance at the contact line and abruptly splash onto the step, but it is often gradual with no noticeable transitions-the fact that boundary conditions have changed is revealed by a (small but measurable) shift in the onset values when the experiment is immediately repeated. For this reason, it was decided in many of the experiments to underfill the container by several millimeters. This created something closer to a 'free' boundary condition which was less susceptible to hysteresis.

Figure 4 shows the time-averaged pattern amplitude and angle (in degrees) as a function of forcing amplitude (in units of the gravitational acceleration $\mathrm{g}$ ), for a $5 \mathrm{~cm}$ deep layer of $5 \mathrm{cSt}$ silicone oil, over the frequency range $50-80 \mathrm{~Hz}$. The crosswise and streamwise wavenumbers, $k_{y}$ and $k_{x}$, are shown in figure 5 as a function of frequency, along with the wavenumber predicted by the inviscid dispersion relation (dashed line). Note that $k_{y}$ matches fairly well with the inviscid prediction, while the full wavenumber is somewhat larger due to the finite streamwise wavenumber $k_{x}$.

In this experiment, the container step is coated with Novec EGC-1700, which is effective at repelling oil, and thus pinning the contact line, at small amplitude. The amplitude, angle and wavenumber are measured by locating the (largest) peak in the Fourier spectrum corresponding to subharmonic waves (in the first quadrant, say). Recall that, due to the evident nonlinearity of the image (see figure 1), this amplitude is not strictly proportional to the subharmonic surface wave amplitude and should be considered as a somewhat qualitative measure. Furthermore, although the amplitude curves in figure 4 suggest supercritical bifurcation, we often observe hysteresis near onset, which is characteristic of subcritical bifurcation. The extent of this hysteresis depends on the frequency used (which determines the detuning) but can also vary at constant frequency due to a dynamic shift in boundary conditions, as mentioned above, if fluid bulges slightly over the step or wets the walls of the container to a greater or lesser extent. The critical forcing is especially sensitive to boundary effects since significant damping can occur at the contact line (Mei and Liu 1973, Hocking 1987, Perlin and Schultz 2000).

Note that, for the 50,60 and $70 \mathrm{~Hz}$ curves in figure 4, there is a fairly sudden transition, signaled by a drop of roughly $10^{\circ}$ in pattern angle and, simultaneously, a more rapid increase in pattern amplitude. The change in surface pattern, which can be striking enough to be seen with the naked eye, occurs nonuniformly as domains of the new pattern invade and supplant the old one. A comparison between prior and subsequent patterns is given in figure 6 . This transition is associated with a decrease in $k_{y}$ and concurrent increase in $k_{x}$. No sudden variation in the boundary conditions is observed at this transition and, as suggested by the 


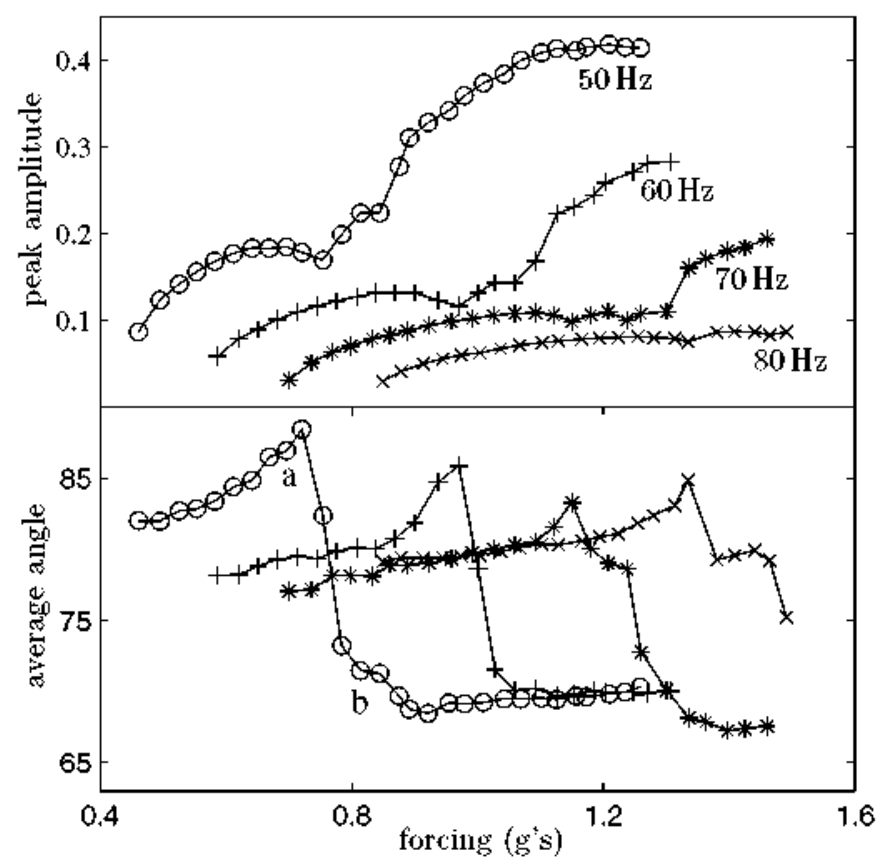

Figure 4. Dependence of pattern amplitude (measured, in arbitrary units, from the subharmonic peak in the Fourier spectrum) and pattern angle (in degrees) on the forcing amplitude (in g's) for a $5 \mathrm{~cm}$ deep container (pinned boundary) of $5 \mathrm{cSt}$ oil.

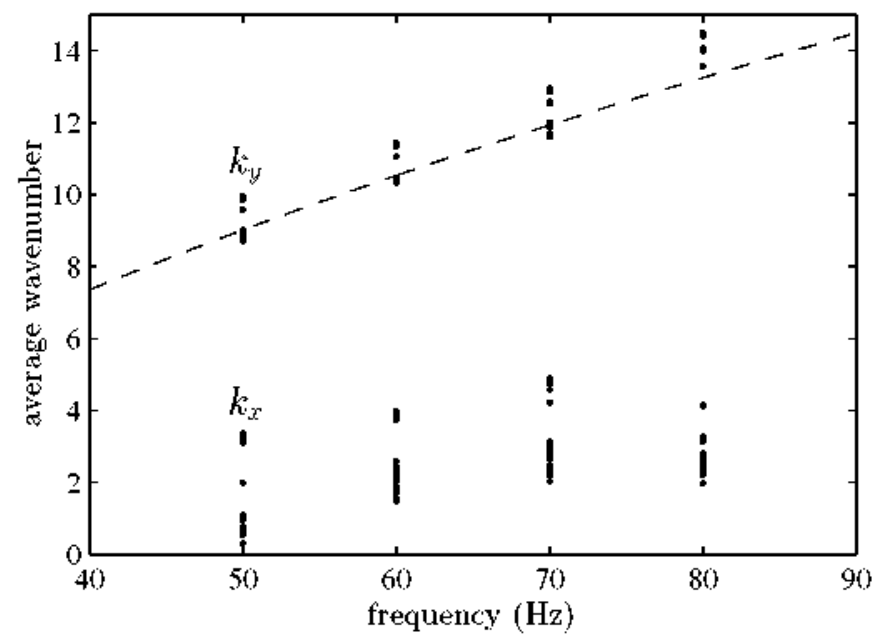

Figure 5. Dependence of pattern wavenumber (in $\mathrm{cm}^{-1}$ ) measured at the forcing amplitudes of figure 4) on forcing frequency for a $5 \mathrm{~cm}$ deep container (pinned boundary) of $5 \mathrm{cSt}$ oil. The dashed curve shows the wavenumber predicted by the inviscid dispersion relation, using a surface tension

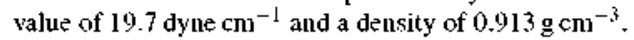

change in $k_{y}$, it is likely related to a (crosswise) mode shift in the pattern triggered by the increase in wave amplitude.

Abrupt transitions do not always occur and, in particular, are not seen if the container is underfilled by several millimeters or more, which allows the contact line to move up and down 


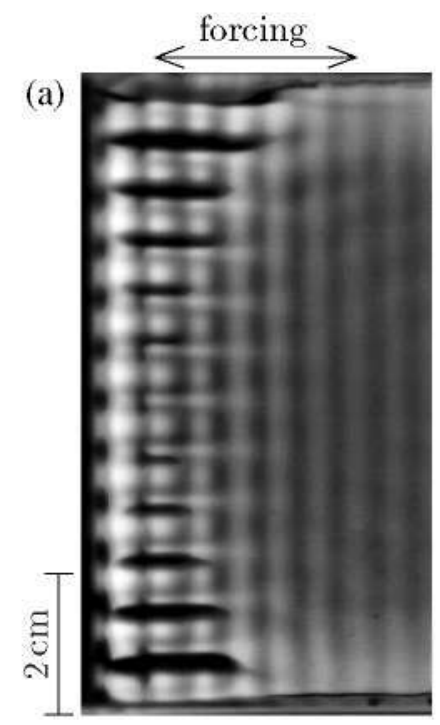

(b)

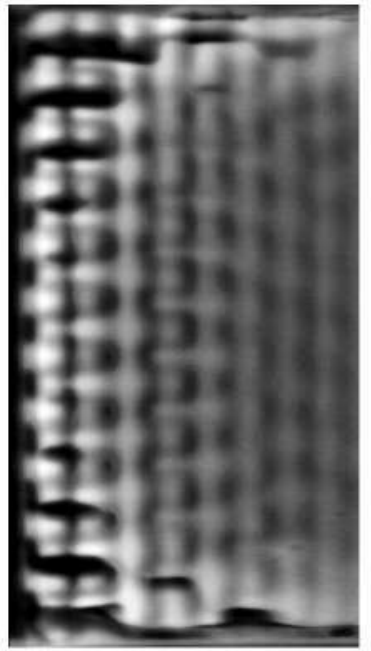

Figure 6. Patterns observed before (a) and after (b) the transition. These correspond to the labeled points on the $50 \mathrm{~Hz}$ curve in figure 4 .

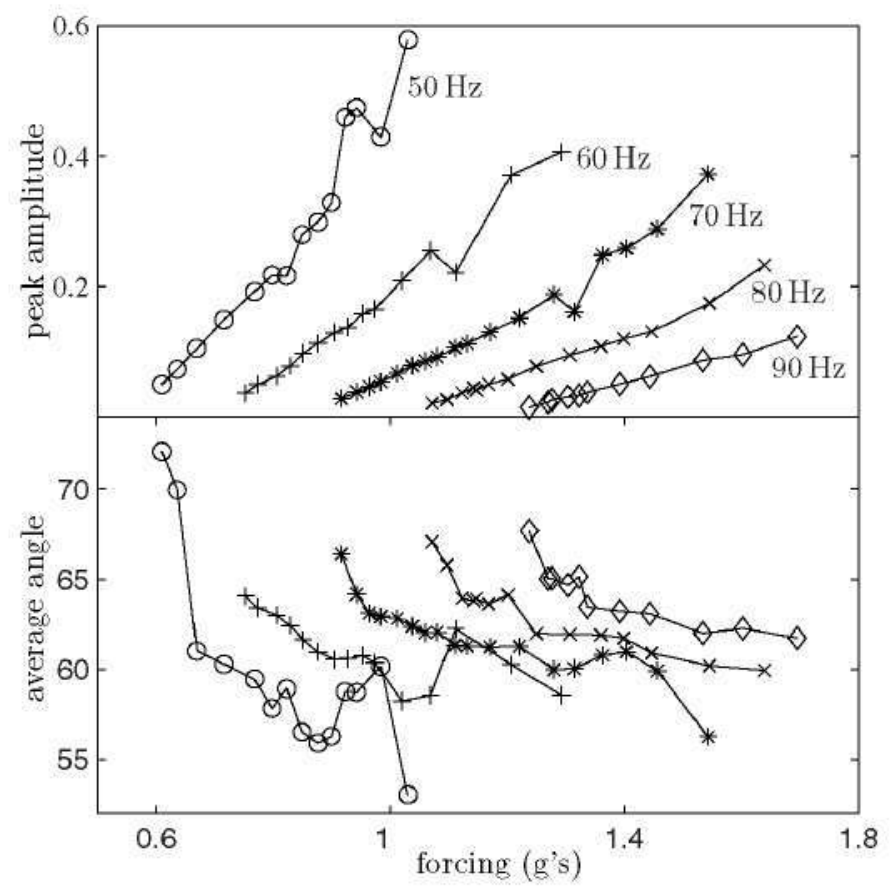

Figure 7. Dependence of pattern amplitude and pattern angle on the forcing amplitude for an underfilled $5 \mathrm{~cm}$ container (wetted boundary) of $5 \mathrm{cSt}$ oil.

the wetted wall. The results for this case are shown in figure 7 . Note that the angle at onset is lower than with pinned boundaries and the system appears to begin directly with the second type of behavior seen in figure 4 (i.e. after the transition). It is not as simple as this, however, because the angles in figure 7 are somewhat lower in general (clustering around $60^{\circ}$ ). 


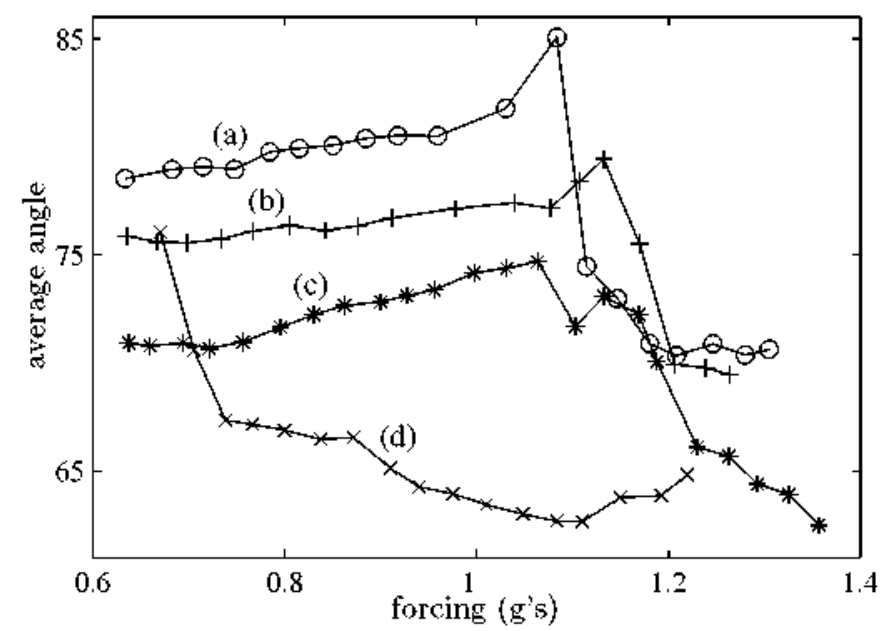

Figure 8. Dependence of the pattern on contact angle. The top curve (a) is obtained with a brimful container of $5 \mathrm{cSt}$ oil vibrated at $60 \mathrm{~Hz}$. while the lower curves (b)-(d) are obtained after successively removing $2 \mathrm{ml}$ of fludd (about $0.5 \%$ of the total volume).

The importance of boundary conditions is further demonstrated in figure 8 , where the time-averaged pattern angle is shown for slightly different filling levels, starting with a flat surface (a) and thrice removing $2 \mathrm{~mL}$ of fluid (b)-(d) to reduce the static contact angle. Each time fluid is removed there is a reduction (on average) in pattern angle.

The lateral boundaries have their own effect on the pattern. The associated boundary layers contribute to damping (Bernoff et al 1989) and select a set of resonant crosswise wavenumbers $k_{y}$. With nearly inviscid waves the selection condition $k_{y}=N \pi / W$ applies where $N$ is an integer and $W$ is the width of the container (see section 4 below). The detuning between the natural frequencies associated with these allowed wavenumbers and the subharmonic resonance frequency can have a significant effect on cross-wave dynamics (Shemer and Lichter 1987, Lichter and Bernoff 1988, Underhill et al 1991). In a real experiment, however, Jones (1984) notes that the detuning 'would be almost impossible to determine' due to its sensitive dependence on channel width. The difficulty is greater in this case because the damping and forcing are considerably higher than in traditional cross-wave experiments (see section 4) and there would be a corresponding shift in the resonance frequencies. Furthermore, a more accurate treatment of the lateral boundary layers would modify the simple wavenumber selection criteria given above. The neutral stability tongues, and hence the resonance frequencies, can be measured directly as in Barnard and Pritchard (1972), for example, but for the high trequencies we use here, damping is large enough to reduce the oscillations of these tongues and make them difficult to resolve; in the numerical model of section 5 (see figure 18) they can be resolved and the effect of detuning can be seen. Since the experiments are close to the large aspect ratio limit ( $N$ is between 20 and 45), and because observing individual resonance tongues directly over this frequency range is beyond experimental resolution, we cannot properly investigate discretization effects in $y$. Although detuning undoubtedly plays a role, it does not seem to be crucial to the patterns that appear at onset. The dispersion relation does a fairly good job of predicting $k_{y}$ (see figure 5) without taking boundary conditions into account, and we see no dramatic effects when changing frequencies (other than minor variations in the extent of hysteresis near onset).

A related concern is that of crosswise mode interactions. It may happen that two crosswise modes ( $N=25$ and $N=26$, for example) are excited simultaneously or nearly 


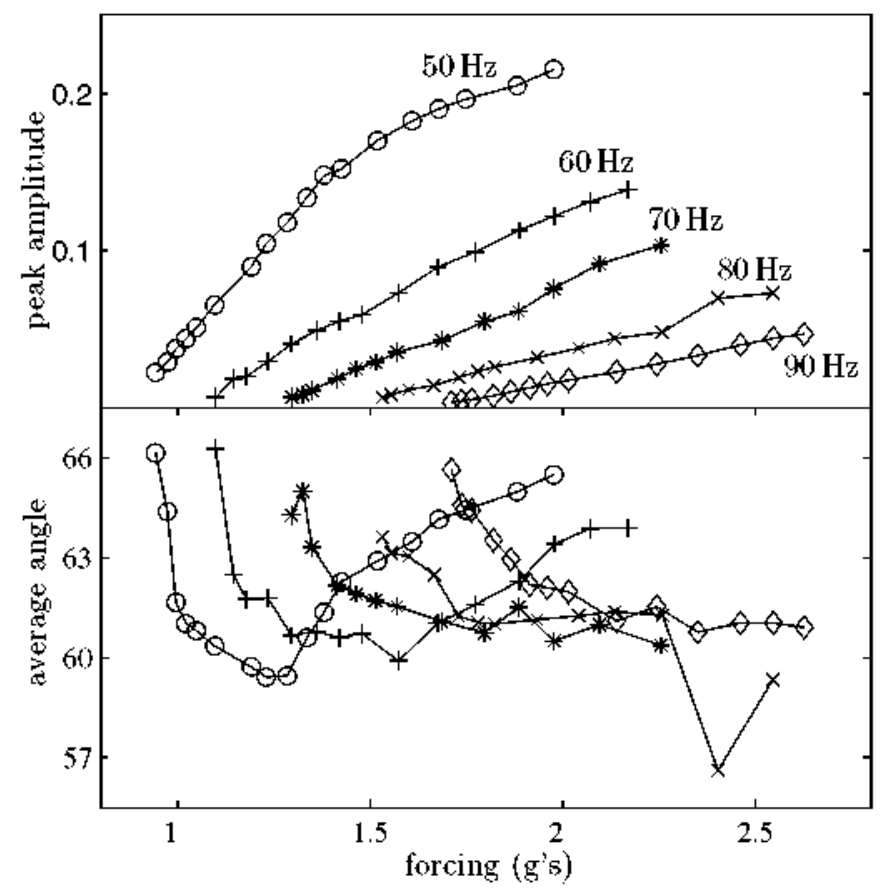

Figure 9. Dependence of pattern amplitude and pattern angle on the forcing amplitude for an undertilled (wetted boundary) $2 \mathrm{~cm}$ deep container of $5 \mathrm{cSt}$ oil.

so. These modes may then interact with each other to produce more complicated dynamics and transitions in the $y$-direction (Lichter and Underhill 1987, Ayanle et al 1990). Indeed, it is likely that this type of crosswise mode interaction is involved in the abrupt transition reported above (figure 6). Modulations in the crosswise direction are also easily seen at higher frequencies (above $100 \mathrm{~Hz}$ ). Across most of the experimental measurements between 40 and $90 \mathrm{~Hz}$, however, and aside from the abrupt transition mentioned, the crosswise wavenumber seems to remain constant-and equal at the two endwalls. Due to the more complicated irregular flow near the lateral boundaries and, especially, in the corners of the container, it is hard to accurately count wavelengths (as can be done at lower frequencies) to check for mode shifting. A Fourier transform cannot resolve neighboring mode numbers well either, so we avoid assigning mode number estimates to the solutions reported in this section.

\subsection{Effect of fluid depth}

The surface wave pattern is much less sensitive to variations in fluid depth than it is to variations in boundary conditions, at least in the regime where the depth is greater than the wavelength of the subharmonic waves. The primary effect of reducing the fluid depth from 5 to $2 \mathrm{~cm}$, for example, is to increase the forcing amplitude required for onset, as shown in figure 9. This is anticipated in the work of Varas and Vega (2007), and can be simply understood: as the depth of the endwall increases it moves a greater volume of fluid with its motion, leading to greater pressure fluctuations at the surface and greater effective parametric forcing. The nature of the selected surface wave pattern, however, is not strongly affected by fluid depth. 


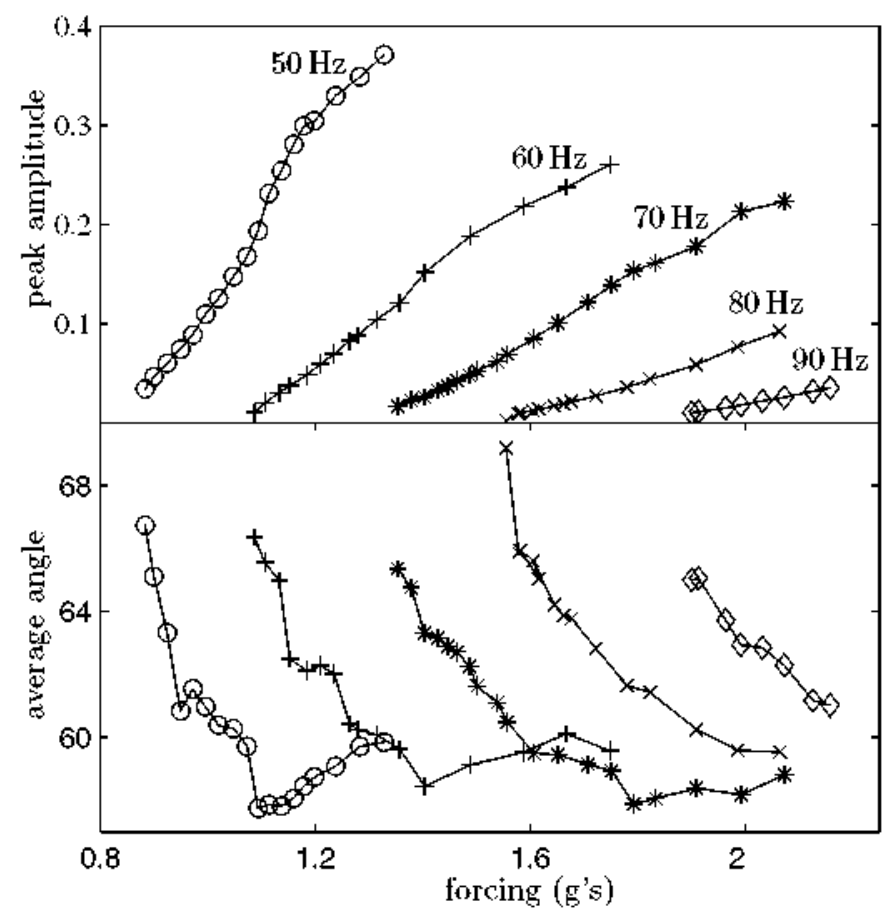

Figure 10. Dependence of pattern anplitude and pattern angle on the forcing anolitude for an undertilled (wetted boundary) $5 \mathrm{~cm}$ deep container of $10 \mathrm{cSt}$ oil.

\subsection{Effect of viscosity}

The effect of increasing viscosity is shown in figures 10 and 11 , obtained with silicone oil of 10 and $50 \mathrm{cSt}$, respectively. These should be compared with figure 7, which is obtained with $5 \mathrm{cSt}$ oil. There is not a lot of difference between the patterns observed with $10 \mathrm{cSt}$ oil (figure 10 ) and $5 \mathrm{cSt}$ oil (figure 7) other than the expected increase in onset values, but the pattern with $50 \mathrm{cSt}$ oil (figure 11) is associated with a drop in average pattern angle. As discussed below and in sections 5.5 and 6 , the increased rotation with higher viscosity seems to be a general feature of these patterns, although the difficulty in properly measuring this angle for highly localized (viscous) patterns makes it hard to establish this quantitatively.

Water has a viscosity of about $1 \mathrm{cSt}$, five times lower than the $5 \mathrm{cSt}$ oil of figure 7 , but patterns obtained with water are not easy to analyze in the manner of that figure. This is due to the strong interaction of the waves generated at opposite walls, both harmonic and subharmonic, which leads quickly to secondary instabilities and spatial disorder. Distinct domains, containing slightly rotated patterns, are a common feature. Measurements of the average pattern angle are, consequently, less meaningful. Nonetheless, the qualitative nature of the pattern is the same as with oil, as illustrated for two different forcing amplitudes in figure 12. The average pattern angle appears to be similar to that seen with $5 \mathrm{cSt}$ oil.

\subsection{Discussion of experimental results}

The experimental results establish that, over a wide range of parameters, horizontally forced subharmonic "cross-waves' do not actually orient themselves crosswise to the motion of the 


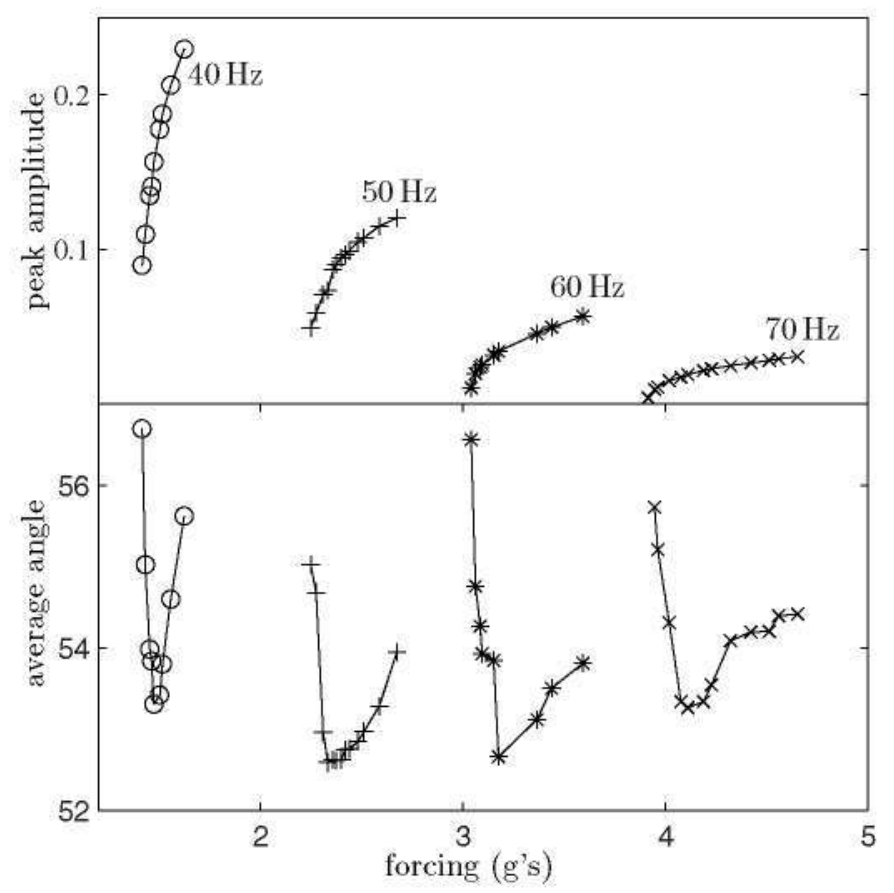

Figure 11. Dependence of pattern amplitude and pattern angle on the forcing amplitude for an underfilled (wetted boundary) $5 \mathrm{~cm}$ deep container of $50 \mathrm{cSt}$ oil.

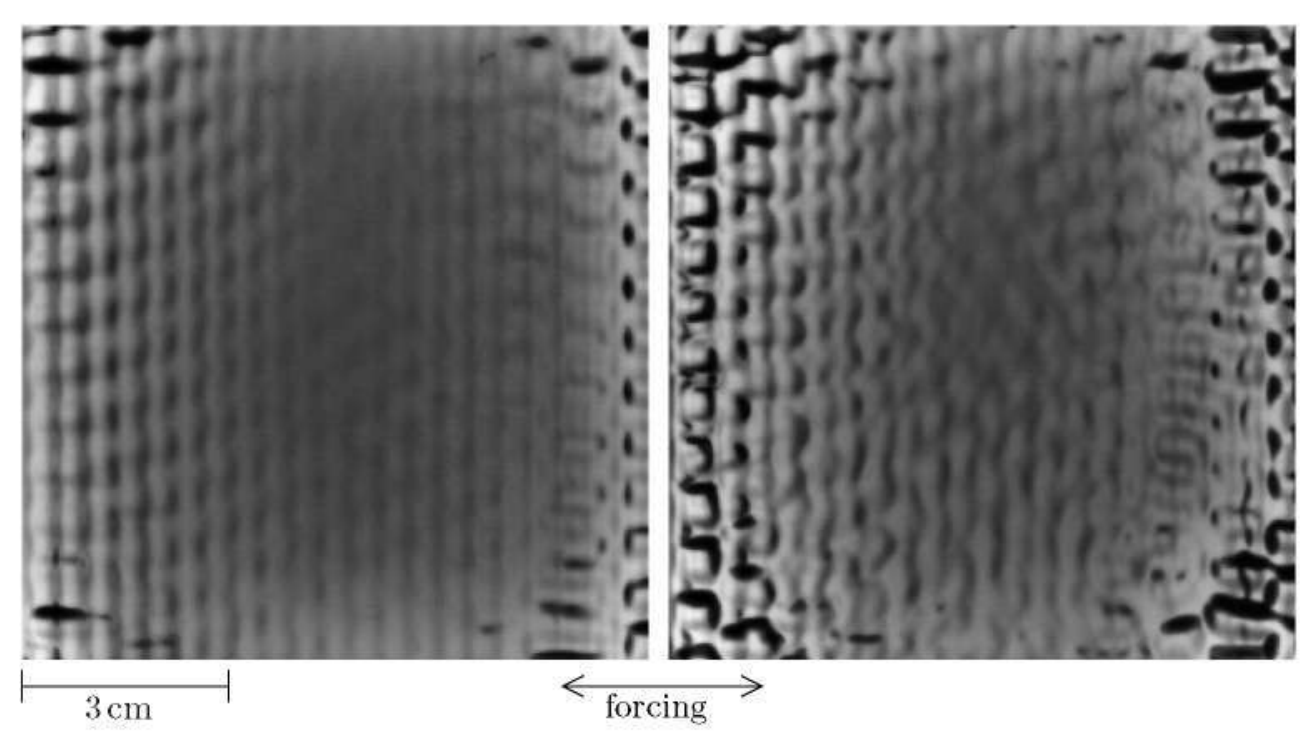

Figure 12. Patterns obtained with water (slightly underfilled) in a $5 \mathrm{~cm}$ container at $80 \mathrm{~Hz}$. Left: a stable pattern with an average angle of about $70^{\circ}$. Right: an irregular pattern obtained with a slightly higher forcing amplitude.

endwall or wavemaker. In fact, for the range $40-100 \mathrm{~Hz}$, over which we are best able to get reliable data in the $9 \mathrm{~cm}$ square container, there is little indication of any transition to ideal cross-waves (i.e. to $90^{\circ}$ ). The qualitative features of the pattern, which reflect the dominance of 
subharmonic waves oriented at $60^{\circ}-80^{\circ}$ with respect to the forcing, are surprisingly insensitive to changes in wavelength (forcing frequency), fluid depth, or viscosity, although there is evident sensitivity to boundary conditions. We also observe this same pattern when using a more conventional wavemaker configuration, with the container held fixed and the shaker attached to a partially submerged vertical plate, which suggests that the results do not rely on the fact that the entire container is shaken, but are applicable to a wide class of wavemaker experiments.

The question of why most previous cross-wave experiments did not find these lozengeshaped patterns naturally arises. A partial explanation may lie in the sheer prominence of the ostensibly perpendicular ridges attached to the endwall or wavemaker, which dominate visually over the interior, lower-amplitude portion of the pattern. Also, most prior experiments (Barnard and Pritchard 1972, Lichter and Underhill 1987, Shemer and Lichter 1987, 1990, Lichter and Bernoff 1988, Shemer and Kit 1989, Underhill et al 1991) used narrow channels (relatively low crosswise mode numbers) where lateral boundary effects can play a crucial role. Neither of these points, however, can explain large-aspect-ratio results such as those of McGoldrick (1968), where a substantial number of wavelengths is visible with no indication of lozenge-shaped structures. It may be that the relatively shallow wedgeshaped vertically oscillating wavemaker used in McGoldrick (1968) produces a significantly different local flow, and hence forcing mechanism, than the horizontally vibrating endwall of our container. The importance of the forcing mechanism is clear when comparing the results of McGoldrick (1968) with those of Taneda (1994) (see also Taneda 1995), where a circular cylinder was half-submerged in water and vibrated vertically at frequencies between 20 and $60 \mathrm{~Hz}$. Although the forcing mechanism is very different in Taneda (1994) and than in section 3 and leads to varying cross-wave amplitude and wavelength along the length of the cylinder, it is apparent that similar oblique patterns consistently arise in that system (in particular, for frequencies near $30 \mathrm{~Hz}$ ), whereas they do not in the experiments of McGoldrick (1968) at comparable forcing frequencies. Qualitatively similar cross-wave patterns with varying or angled crests were also observed in connection with secondary instabilities that produce slow temporal modulations; see, e.g. figure 4 of Underhill et al (1991).

Much of the explanation for the patterns found in section 3 certainly lies with the range of frequencies used and the size of the dimensionless damping $\gamma=2 v k^{2} / \omega$. For example, the damping in our experiments is generally higher (by a factor of 10 or so; see table 1 below) than in McGoldrick (1968), where water was vibrated at frequencies of $32 \mathrm{~Hz}$ or less. Despite the relatively weak dependence on viscosity found here, it is possible that a transition to ideal cross-waves occurs at still lower values, where most previous cross-wave experiments lie.

Several possible selection mechanisms for the particular pattern (angle) we see suggest themselves. It is tempting, for instance, to assume that the ideal angle is $60^{\circ}$ and due to a nonlinear preference for hexagons. Alternatively, the angle might be selected by a resonant triad interaction (two subharmonic waves with one harmonic wave) or a five-wave resonance (four subharmonic waves with one harmonic wave). Such resonances predict angles between about $50^{\circ}$ and $75^{\circ}$, depending on frequency, for the experiments reported above. However, nonlinear selection mechanisms should come into play at finite amplitude, while we find the same basic pattern at onset and beyond, at least when boundary conditions can be assumed constant. This suggests a linear selection mechanism wherein these patterns reflect the critical linear eigenfunctions of the problem.

The issues identified above can be clarified with model equations and numerical simulations, which provide both greater detail and parameter variability. In the following section we begin by looking carefully at the existing theoretical models used to simulate 
Table 1. Range of container size (length $\times$ width $\times$ depth) and fotcing parameters considered in previous cross-wave experiments, compared with the experiments teported hete (final row). The * indicates that the mode number is estimated.

\begin{tabular}{lcccc}
\hline & & & & Surface \\
Experiment & $L \times W \times D$ & $\mathrm{~Hz}$ & $\mathrm{~N}$ & $\begin{array}{c}\text { Container size }(\mathrm{cm}) \\
\text { damping } \\
\gamma \times 10^{4}\end{array}$ \\
\hline McGoldrick (1968) & $300 \times 61.4 \times 44$ & $6.7-32$ & $9-85^{\star}$ & $1-19$ \\
Barnard and Ptitchard (1972) & $270 \times 30.6 \times 16$ & $3.1-10.4$ & $1-10$ & $0.11-3.2$ \\
& $120 \times W \times 30$ & & & \\
Lichter and Underhill (1987) & $11.37 \geqslant W \geqslant 29.15$ & 10 & $3-9$ & $2.2-3$ \\
Shemer and Lichter (1987) & $1800 \times 120 \times 60$ & $3.45-3.65$ & 5 & $0.15-0.16$ \\
Shener and Kit (1989) & $1800 \times 120 \times 60$ & $2.3-3.6$ & $2,3,5$ & $0.038-0.15$ \\
Shemet and Lichter (1990) & $1800 \times 120 \times 60$ & $2.8-4.3$ & $3,5.6,7$ & $0.07-0.25$ \\
Lichter and Bernoff $(1988)$ & $120 \times 30.97 \times 27.2$ & $7.6-8.16$ & 6 & $1.4-1.6$ \\
Undethill $e t$ al $(1991)$ & $92 \times 30.9 \times 25.8$ & $7-9$ & $5-7$ & $1.2-1.8$ \\
Section 3 & $9 \times 9 \times(2-5)$ & $40-100$ & $20-45$ & $200-3800$ \\
\hline
\end{tabular}

cross-wave dynamics, which are based on a nonlinear Schrödinger (NLS) equation first derived by Jones (1984), and their applicability to the current experiment.

\section{Comparison with existing work}

The table below illustrates the range of parameters investigated in a selection of previous cross-wave experiments, for comparison with those of section 3 (the final row of the table). The mode number $N$ is defined as the number of half-wavelengths spanning the container; the crosswise wavenumber satisfies $k_{y}=N \pi / W$ where $W$ is the width of the tank. The final column shows the dimensionless surface damping coetificient $2 v k_{y}^{2} / \omega$, which is one measure of departure from the inviscid limit. As the table illustrates, these results apart from (some of) McGoldrick's lie in the nearly inviscid gravity wave regime. They are obtained in large wave tanks with low mode numbers. The dimensionless surface damping is very small: of the order of $10^{-4}$ or less. Of course, the surface is not the only source of damping. Dissipation at the sidewalls is very important for experiments with low wavenumbers (modes) and wavemaker dissipation plays a role in nearly all cases. Depending on the depth of the container, dissipation at the bottom boundary can be significant as well. For example, in the experiments of Barnard and Pritchard (1972) (see second row of table 1), estimates based on the formulae of Bernoff et al (1989) (see below) suggest that dissipation at the bottom is negligible for $N>1$ while the dissipation at the sides dominates for $N \leqslant 6$. The dissipation at the wavemaker is important and comparable to that at the surface. In the experiments of section 3 , the wavemaker dissipation is the only significant contribution aside from the surface damping (again, they are comparable). These other sources of damping are important, and contribute to the difficulty of accurately calculating damping in a real experiment-contact line motion, meniscus effects, and finite-amplitude processes like wave breaking, can also significantly increase damping. We do not include estimates of additional damping coefficients in table 1, but doing so would not change the main point: that the damping in our experiments is higher (by several orders of magnitude) than the damping in most previous cross-wave experiments. The experiments described in section 3 lie in a distinct and relatively unexplored regime: higher frequency and low to moderate damping $(0.02 \lesssim \gamma \lesssim 0.38)$. 


\subsection{Theory: subharmonic instability and the NLS equation}

While a number of early experiments (Schuler 1933, Lin and Howard 1960) added to the observations of Faraday (1831), a proper theoretical understanding of cross-waves began with Garrett (1970), who showed that the cross-wave instability could be described by Mathieu's equation, and thus exemplifies parametric instability, just as the vertically forced Faraday wave instability does. The difference is that the energy needed to excite crosswaves is provided by the wavemaker (more generally, a moving boundary) and is therefore not distributed uniformly in space. Garrett found good agreement between his theory and the experiments of (Lin and Howard 1960) particularly with respect to the rightward shift of the frequency-amplitude curves. He emphasized in his conclusion that "cross-waves are a transverse instability independent of the primary motion' (Garrett 1970). This may be contrasted with an earlier theory put forth by McGoldrick (1968) where the parametric forcing mechanism was assumed to be provided by the primary (harmonic) wave field. McGoldrick likewise obtained a Mathieu-type equation (distinct from that of Garrett) which predicted subharmonic instability tongues and, after damping was included in an approximate way, onset values comparable with those measured in his experiment. The experiments of McGoldrick were performed in a tank of large aspect ratio and were initiated by a vertically oscillating wedge-shaped plunger that, judging from the figures provided, penetrates just a few centimeters below the fluid surface (less than the wavelength of most of the subharmonic waves considered). This is unlike most other wavemaker experiments where a moving flap extends well below the surface and thus moves a larger volume of fluid. It may be that with the relatively shallow wavemaker of McGoldrick, the primary harmonic waves do provide the mechanism for promoting cross-waves, but it is clear from subsequent theory (Jones 1984, Bernoff et al 1989, Varas and Vega 2007) that the harmonic surface waves, in most cases, lead only to minor corrections in the description of the cross-wave instability. The primary forcing mechanism comes from the evanescent harmonic flow field that is established near the wavemaker by its motion and not the harmonic surface waves that radiate away from it.

The theory of cross-waves was advanced by Jones (1984), who obtained a cubic NLS equation to describe the slow spatial and temporal evolution of the cross-wave amplitude $A(x, t)$. This equation was subsequently augmented by different authors to include viscous effects (Lichter and Chen 1987, Bernoff et al 1989, Shemer and Kit 1989) and mode interactions (Ayanle et al 1990). These theoretical models were compared successfully to experiment (Lichter and Bernoff 1988, Kit and Shemer 1989, Shemer and Kit 1989, Underhill et al 1991) and capture many features of cross-wave behavior. However, they rely on certain assumptions, several of which do not apply to the experiments of section 3 . These assumptions are:

(i) Damping is assumed to be small or nonexistent.

(ii) Forcing is assumed to be small so that an asymptotic expansion in a related small parameter can be made.

(iii) Surface tension is ignored (since most cross-wave experiments involve gravity waves) except perhaps in locating the resonance frequencies (Lichter and Bernoff 1988, Shemer and Lichter 1990).

(iv) The downstream variation of the subharmonic waves is assumed to be much slower than the variation in the crosswise $y$ direction $\left(A_{x} \ll k_{y} A\right)$, i.e. the waves are assumed to be oriented crosswise.

(v) The downstream variation of the subharmonic waves is assumed to be much slower than the variation of the evanescent flow field that drives them. 
The final assumption is particularly important because if $A(x, t)$ varies slowly in $x$, on a different lengthscale than the localized flow generated by the motion of the wavemaker, then there is no distributed source in the NLS equation, which describes only the slow spatial scale. The forcing enters via a homogeneous boundary condition. This situation is in contrast both to the case of vertically forced Faraday waves (where the forcing is uniformly distributed) and the theory of Varas and Vega (2007) where the forcing term appears in the governing equation because it varies on the same scale as the modulation (as in the experiment of section 3 ).

To examine the consequences of these underlying scaling assumptions we utilize the NLS model derived by Bernoff et al (1989). This version is particularly useful for the comparison made here because it explicitly includes the leading order effects of viscous damping at the wavemaker, side walls, bottom and free surface. The formulation assumes a semi-infinite channel (so complications due to interaction of the two sides can be ignored) and a planar wavemaker, which is analogous to the flat endwall of our experimental container.

The NLS model obtained by Bernoff et al (1989) is parameterized by three dimensionless quantities: the nondimensional forcing $\epsilon=a k_{y} \ll 1$, the Reynolds number $\operatorname{Re}=\sigma /\left(v k_{y}^{2}\right)$, and the nondimensional depth $d=k_{y} D$. Here $a$ and $\sigma$ are the amplitude and (half) frequency of the endwall displacement $a \cos (2 \sigma t)$ and $v$ is the kinematic viscosity. $D$ is the depth of the fluid, $W$ is the width of the container and $k_{y}$ is the crosswise wavenumber: $k_{y}=N \pi / W$. The rescaled equations can be written as

$$
\begin{aligned}
& i A_{T}=A_{X X}+(\Lambda-i) A \pm A|A|^{2}, \\
& A_{X}=-\tilde{R} \bar{A}-\tilde{D} A \text { at } X=0 \\
& A \rightarrow 0 \quad \text { as } X \rightarrow \infty
\end{aligned}
$$

in terms of a slow time $T=\epsilon_{c}^{2} \sigma t$ and a slow spatial coordinate $X=\left(2 \epsilon_{c} / \sqrt{S}\right)\left(k_{y} x\right)$. The small quantity $\epsilon_{c}$ characterizes the internal viscous scale:

$$
\epsilon_{c}=\left\{\frac{2}{\operatorname{Re}}+\frac{1}{\sqrt{2 \operatorname{Re}}}\left[\frac{1}{\sinh (2 d)}+\frac{1}{N \pi}\left(1-\frac{2 d}{\sinh (2 d)}\right)\right]\right\}^{1 / 2}
$$

and gathers contributions from the free surface, bottom and side walls, respectively. The dimensionless forcing and wavemaker damping are given by $\tilde{R}=\epsilon\{d[3 \tanh (d)+$ $1 / \tanh (d)]-2\} /\left(2 \epsilon_{c} \sqrt{S}\right)$ and $\bar{D}=(1-i)\left(\epsilon_{c} \sqrt{2 S \operatorname{Re}}\right)$, respectively, with $S=1+$ $2 d / \sinh (2 d) . \Lambda$ is a detuning parameter which, after neglecting the spatially dependent contribution from the harmonic surfaces waves (Bernoff et al 1989), is given by

$$
\Lambda=\frac{1}{\epsilon_{c}^{2}}\left[\frac{\sigma-\sigma_{0}}{\sigma_{0}}-\frac{2}{\operatorname{Re}}\right]+1 .
$$

The first term captures the relative deviation of (half) the forcing frequency $\sigma$ from the resonant frequency $\sigma_{0}=\sqrt{g k_{y}}$ while the rest is due to viscous effects.

Linearizing (2)(a) and looking for stationary states $A(X, T)=A_{0} \mathrm{e}^{k X}$ gives the neutral stability curve for cross-waves, which can be expressed as

$$
\tilde{R}^{2}=\sqrt{\Lambda^{2}+1}+|\tilde{D}|\left[\sqrt{2}\left(\kappa_{r}+\kappa_{i}\right)+|\tilde{D}|\right]
$$

where $\kappa=\kappa_{r}+i \kappa_{i}=\sqrt{i-\Lambda}$ with $\kappa_{r}<0$. Restoring dimensions and expressing the critical amplitude in terms of relative acceleration $g_{\text {rel }}=a(2 \sigma)^{2} / g$ where $g$ is the acceleration of 


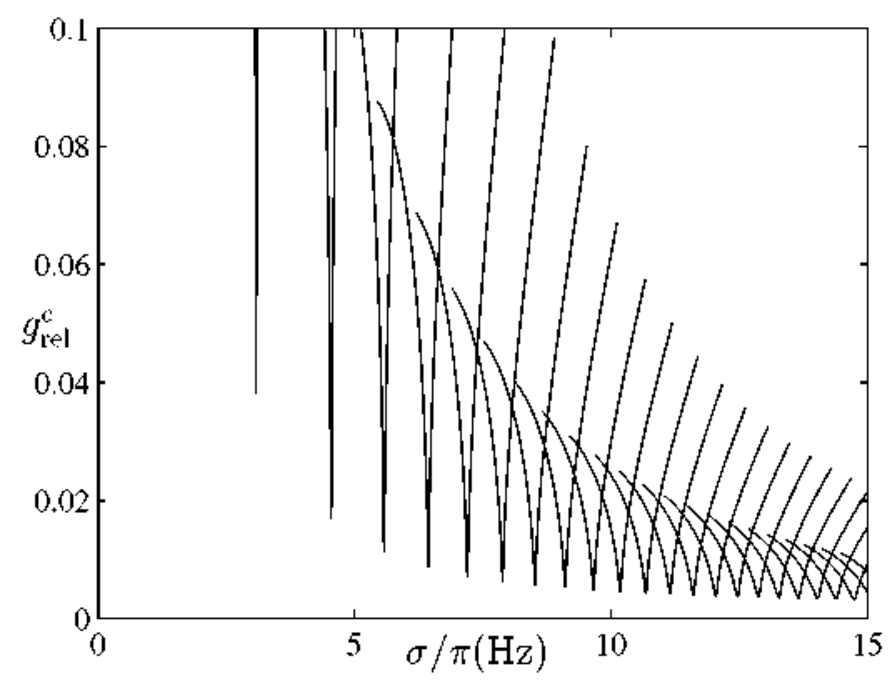

Figure 13. Neutral stability curves corresponding to (6) with $v=1 \mathrm{cSt}, W=30 \mathrm{~cm}$ and $D=$ $15 \mathrm{~cm}$. Note that the minimum values are monotonically decreasing with frequency (mode number).

gravity, we obtain

$$
g_{\text {rel }}^{c}=\frac{8 \sigma^{2} \epsilon_{c}}{g k_{y} R \sqrt{S}}\left\{\sqrt{\Lambda^{2}+1}+|\bar{D}|\left[\sqrt{2}\left(\kappa_{r}+\kappa_{i}\right)+|\bar{D}|\right]\right\}^{1 / 2} .
$$

Equation (6) produces neutral stability curves like those shown in figure 13. In that figure we take $v=1 \mathrm{cSt}, W=30 \mathrm{~cm}$ and $D=15 \mathrm{~cm}$, which are close to the parameters used in Barnard and Pritchard (1972), Lichter and Bernoff (1988) and Underhill et al (1991) if the wavemaker depth of $14.1 \mathrm{~cm}$ is substituted for $D$ in the two more recent experiments, and plot the interval $\left[\sigma_{i} / \pi-1, \sigma_{0} / \pi+1\right]$ for modes numbers $N=1, \ldots, 21$. Note the decrease in successive minima (which occur near the resonant frequencies $\omega_{0} / \pi=3.09,4.55,5.58, \ldots$ ) in figure 13. An increase in critical acceleration for resonance tongues with small $N$ is expected because of viscous dissipation at the container bottom, which diverges as $\sigma k_{y} \rightarrow 0$ (the limit $d \rightarrow 0$ in (3)). This behaviour is familiar as well from the study of vertically forced Faraday waves in shallow containers. The interesting thing is that this behaviour persists even if dissipation at the bottom is neglected (the deep layer limit $d \gg 1$ ). To see this clearly from (6) we ignore not only damping at the container bottom but also at the wavemaker $\left(\bar{D}=0\right.$ ) and side walls. Then, setting $\sigma=\sigma_{0}$ and using the gravity wave dispersion relation $\sigma^{2}=g k_{y}$ and the fact that $S \simeq 1$ and $R \simeq 4 k D$ when $d \gg 1$, (6) simplifies to

$$
g_{\text {rel }}^{c} \simeq \frac{2 \sqrt{2 v}}{D}\left[\frac{W}{N \pi g}\right]^{1 / 4} .
$$

Thus $g_{\text {rel }}^{c}$ (evaluated at $\sigma_{0}$ ) decreases monotonically with $N$ even if the container is very deep and the only dissipation comes from the free surface.

This decrease in critical amplitude with mode number (frequency) is in contrast to the case of distributed forcing where a direct balance between damping and forcing occurs. In the case of vertically forced Faraday waves in a deep fluid layer, for instance, the critical acceleration scales as $g_{\text {rel }}^{c} \sim v k \sigma$ (see, e.g. Porter and Silber 2004) and increases 


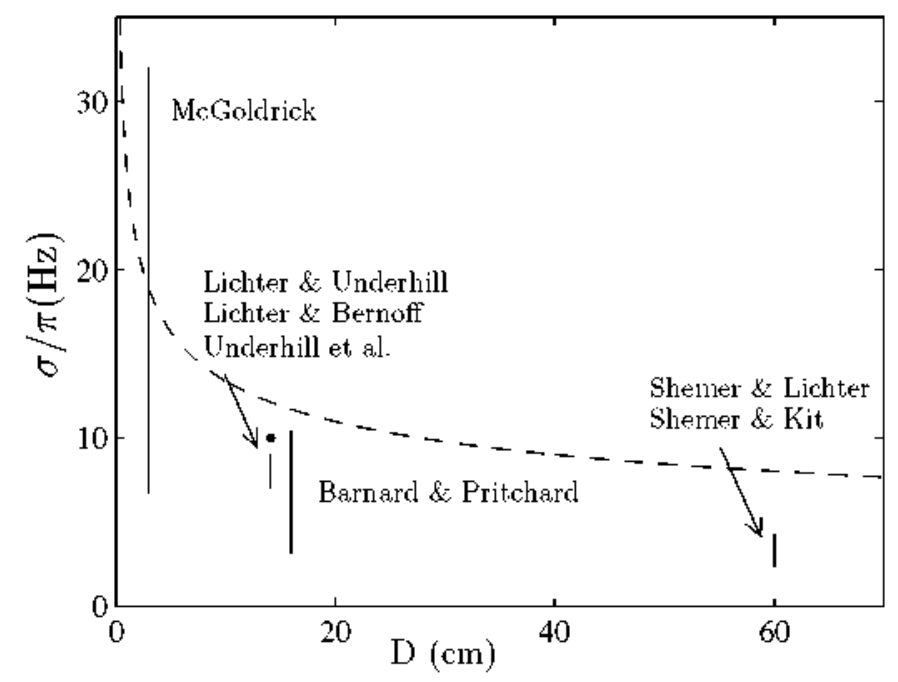

Figure 14. The dashed curves show where the forcing frequency $\sigma / \pi$ reaches $\left[g^{4} /\left(4 D^{2} \nu\right)\right]^{1 / 7 / \pi}$ as a function of (wavemaker) depth $D$ for $v=1 \mathrm{cSt}$. The range of frequencies considered in the experiments of table 1 are shown as vertical lines.

monotonically with $\sigma$ (or $k$ ). A similar balance holds in the equations derived in Varas and Vega (2007) for the case of distributed but nonuniform forcing (note the nearly linear relation displayed in figure 14 of that reference). The key is the final scaling assumption mentioned above. In the NLS equation the forcing enters via the boundary condition, where it induces a 'slow' downstream variation $A_{X}$ characterized by a complex wavenumber $\kappa$. It is the square of this wavenumber (and thus the square of the forcing), via the dispersive term $A_{X X}$, that must balance damping. With no dissipation at the container bottom the decrease in critical acceleration values with increasing mode number (and hence increasing surface damping coefficient $\gamma$ ) is somewhat counterintuitive and warrants carefil examination.

There are several cross-wave experiments where onset values can be quantitatively compared across distinct modes and we summarize these below.

McGoldrick (1968) measured marginal stability curves for $N=24$ and $N=25$ (wavelengths of 5.12 and $4.91 \mathrm{~cm}$, respectively, in a container of width $W=61.4 \mathrm{~cm}$ ) but these modes are so similar that no significant difference between their onset values can be seen.

Neutral stability curves are provided for modes $N=2$ and $N=3$ in Barnard and Pritchard (1972) (figure 2). Critical values of $0.087 \mathrm{~g}(\mathrm{~N}=2)$ and $0.072 \mathrm{~g}(\mathrm{~N}=3)$ can be estimated, and this decrease is consistent with the NLS model and the behaviour shown in figure 13. However, the fact that the wavelengths in question $(30.6$ and $20.4 \mathrm{~cm})$ are greater than the depth of $16 \mathrm{~cm}$ means that this decrease is likely due to the change in damping at the container bottom.

Onset values are measured for modes $N=5$ and $N=7$ in Underhill et al (1991) (figures 3 and 7), providing estimates for the critical forcing of $0.064 \mathrm{~g}(\mathrm{~N}=5)$ and $0.074 \mathrm{~g}$ $(N=7)$. The fluid depth of $25.8 \mathrm{~cm}$ is greater than the wavelengths of these modes (12.36 and $8.83 \mathrm{~cm}$, respectively), which puts the experiment close to the deep layer limit. The measured increase in onset with increasing mode number is not explained by the NLS model, but is consistent with waves parametrically excited (in deep containers) by distributed forcing, like vertically forced Faraday waves or the two-dimensional horizontally shaken system considered in Varas and Vega (2007). 
Neutral stability curves for modes $N=3,5,6$ and 7 were measured in Shemer and Lichter (1990) (figure 2), providing (minimum) onset values of $0.153,0.137,0.144$ and $0.167 \mathrm{~g}$, respectively. The wavelengths of these modes $(80,48,40$ and $34.3 \mathrm{~cm})$ should be compared to the depth of $60 \mathrm{~cm}$. Note that, except for the transition from $N=3$ (where the wavelength exceeds the container depth) to $N=5$, the onset value increases with increasing mode number. Again, this is not explained by the NLS model, although this aspect of the experiment is harder to interpret than in Underhill et al (1991) because of the segmented wavemaker used, which generates larger dissipation via vortex shedding at the discontinuities (Shemer and Lichter 1990).

Our own experimental set-up is designed for higher frequencies but we have measured onset values for gravity waves with mode numbers $N=7, \ldots, 10$ over the range $10-14 \mathrm{~Hz}$ in a larger $20 \mathrm{~cm}$ square container with a $3 \mathrm{~cm}$ deep layer of $10 \mathrm{eSt}$ silicone oil, obtaining 0.138 , $0.149,0.164$ and $0.175 \mathrm{~g}$, respectively. The increase in onset with frequency (mode number) is clear and, again, suggests a limitation of the NLS model.

We were primarily interested in section 3 not with onset values, but with measuring the downstream modulation of the observed patterns. The NLS model does, of course, predict modulation via the envelope $A(x, t)$ (see, e.g. the simulations of Lichter and Chen 1987), but this must remain small for self-consistency. The modulation in $x$ should be much slower than the crosswise modulation $\left(A_{x} \ll k_{y} A\right)$ and the variation of the forcing (evanescent flow field). The latter requirement can be problematic since the extent of the forcing scales with the depth $D$ of the wavemaker (Bernoff et $a l 1989$, Varas and Vega 2007), which in a typical experiment is relatively large. One self-consistency test of the NLS model is the requirement $k_{x} D \ll 1$ where $k_{x}$ (proportional to the imaginary part of $\kappa$ ) is used to characterize the modulation of $A(x, t)$. From the solution of the linearized problem in Bernoff $e$ al $(1989)$ we have $k_{x}=2 \kappa_{i} \epsilon_{c} k_{y} / \sqrt{S}$. This quantity depends on all the parameters of the problem, including mode number, but may be simplified by assuming a deep container $(d \gg 1)$, no detuning, and negligible damping at the wavemaker, sides and bottom. With these simplifications

$$
k_{x} \simeq 2 \sqrt{\frac{\nu}{\sigma}} k_{y}^{2},
$$

which tends to zero as $v \rightarrow 0$ yielding pure cross-waves, as expected. The condition $k_{x} D \ll 1$ can then be transformed to a condition on the frequency: $\sigma \ll\left[g^{4} /\left(4 D^{2} v\right)\right]^{1 / 7}$. Figure 14 shows the curve where equality holds along with the range of forcing frequencies used in the experiments of table 1. Most previous cross-wave experiments lie below this line (the exception being McGoldrick 1968) but not always comfortably so-in the experiments of Barnard and Pritchard (1972), for example, this estimate for $k_{x} D$ reaches values of about 0.6 . Furthermore, (8) is only a simplified estimate that, among other things, ignores detuning, which is known to greatly affect the modulation of $A(x, t)$. In Shemer and Kit (1989), for example, where the condition $k_{x} D \ll 1$ holds easily, the separation of scales is not maintained throughout. The authors find that 'the gradual reduction of the frequency of forcing was accompanied by an increase of the wave amplitude at the wavemaker, while the extent of the region with noticeable cross-wave amplitude decreased, so that the whole wave field became restricted to the vicinity of the wavemaker'. These localized solutions decay on the same lengthscale as the localized harmonic flow near the wavemaker (i.e. on the scale of the wavemaker depth) and, thus, are not expected to be well described by the NLS model.

In summary, the various NLS models that followed (Jones 1984) were successful in a number of ways. They show the kind of slow soliton-like modulations observed in many experiments (Barnard and Pritchard 1972, Lichter and Shemer 1986, Underhill et al 1991). They capture the effect of detuning on a particular mode, like the transition from supercritical 
to subcritical bifurcation (Lichter and Bernoff 1988), and even the complications due to mode interaction (Ayanle et al 1990). The NLS models, however, represent a particular balance between forcing and damping that does not hold when the localized forcing region cannot be considered small, as revealed by the increase in onset value with mode number observed in our experiment and, to some extent, in Underhill et al (1991) and Shemer and Lichter (1990). The underlying assumption of the NLS models of a separation of lengthscales in $x$, with the modulation allowed to vary only slowly compared to the localized flow field set up near the wavemaker, can restrict their applicability to very small values of damping (alternatively, very low frequencies) or to relatively shallow wavemakers.

The experiments we describe in section 3 are well beyond the regime where the NLS model is justified, even if extended to include surface tension. Damping and forcing are only moderately small ( $\gamma$ is less than 1 , but not by several orders of magnitude; see table 1), not nearly small enough to support the scaling assumptions required by the NLS model. In no sense is there a separation of lengthscales in the $x$-direction. On the contrary, in our experiments, the lengthscale of the forcing helps determine the lengthscale of downstream variation for the subharmonic waves. Subharmonic waves persist where the forcing supports them and quickly decay outside of this localized region. Their spatial variation and the variation of the underlying flow that provides the forcing are linked. Because this downstream variation is not slow, the patterns deviate strongly from ideal cross-waves, as described in section 3 .

\section{Numerical simulations}

For the reasons discussed in section 4 , we cannot use the NLS model to simulate our experiments, and must take a different approach. We require a model that permits a large variety of two-dimensional patterns (i.e. that does not assume proximity to ideal cross-waves a priori) and that incorporates a spatially extended forcing term (i.e. with no separation of lengthscales in $x$ ). Furthermore, it should work well not only with very low but also with moderately low damping (characteristic of low viscosity silicone oils and trequencies of $50-100 \mathrm{~Hz}$ ). Such a model, to our knowledge, does not yet exist for the case of horizontal forcing, and it is beyond the scope of the current paper to derive one (this will be the subject of future work). The required features, however, are identical to those needed to model recent vertically forced Faraday wave experiments (see, for example, Arbell and Fineberg 2002) and a good choice for this purpose is the model derived by Zhang and Vinals (1997). This wellknown model captures the weakly nonlinear dynamics of parametrically driven surface waves in weakly damped, deep containers. It reduces the three-dimensional Navier Stokes equations to a (nonlocal) set of equations involving only the surface coordinates $x=(x, y)$, and thus greatly facilitates computations.

The use of this particular model in the context of our experiments can address some questions, but not others. The restriction to deep fluid layers is not a significant limitation since the depth of the experimental container, even in the $2 \mathrm{~cm}$ case, is at least several times greater that the wavelength of the subharmonic waves (less than $1 \mathrm{~cm}$ at forcing frequencies of $40 \mathrm{~Hz}$ or more). The restriction to weak damping is a concern, but significant error is expected only for the $50 \mathrm{cSt}$ oil experiments. The nondimensional damping factor $\gamma$ ranges from 0.022 (at $40 \mathrm{~Hz}$ ) to $0.04 \mathrm{l}($ at $100 \mathrm{~Hz}$ ) for $5 \mathrm{cSt}$ oil and from $0.045($ at $40 \mathrm{~Hz}$ ) to 0.082 (at $100 \mathrm{~Hz}$ ) for $10 \mathrm{cSt}$ oil. For most purposes, these values can be considered small in the Zhang-Viñals model (Skeldon and Porter 2011). Only for $50 \mathrm{cSt}$ oil does $\gamma$ exceed 0.1 . With oil of this viscosity $\gamma$ is large enough $(0.4$ at $100 \mathrm{~Hz}$, for example) that the ZhangViñals equations are not expected to apply. More significantly, this model assumes an 
infinitely extended fluid layer and cannot capture boundary effects (contact line dynamics, boundary layer damping, etc) that are important in experiments. Finally, since the forcing term used by Zhang and Viñals describes vertical forcing, it must be modified to apply to the horizontally forced case. As described below, the modification we make relies on the theoretical work of Varas and Vega (2007), which shows how horizontal forcing leads to a parametric forcing mechanism that is completely analogous to the parametric forcing of the vertically forced case, except in its spatial dependence. The spatial profile of this parametric forcing, explicitly obtained in Varas and Vega (2007), is used here.

It is clear that this model cannot be expected to be in quantitative agreement with the experiments, not least because it neglects damping at the container walls and contact line. The purpose of the simulations in this section is to qualitatively test the key idea that horizontal forcing in a sufficiently deep container leads to a spatially dependent, but extended, parametric forcing mechanism, strongest near the walls, that is otherwise analogous to uniform (vertical) parametric forcing. The resulting picture of subharmonic patterns largely determined by the spatial variation of an extended forcing function (not driven by a boundary condition) is qualitatively different from the picture provided by the NLS model. The fact that a simplified model with only this forcing mechanism, neglecting all damping at the boundaries, is still in reasonable agreement with the experimental results of section 3 and immediately reproduces similar surface wave patterns is a strong argument for the validity of this idea and for the generic nature of these patterns. It demonstrates, for one thing, that they cannot be dismissed as the product of experimental imperfections or peculiar boundary layer effects. Similar rotated patterns must be expected in vibrated fluid systems (of comparable scale and damping) whenever that vibration generates localized parametric forcing.

\subsection{The modified Zhang-Vinals model}

The model derived by Zhang and Viñals (1997) for the case of weakly damped parametrically excited surface waves in deep containers is

$$
\begin{aligned}
& \left(\partial_{t}-\gamma \nabla^{2}\right) h-\hat{D} \Phi=N_{1}(h, \Phi), \\
& \left(\partial_{t}-\gamma \nabla^{2}\right) \Phi-\left[\Gamma_{0} \nabla^{2}-G_{0}+G(t)\right] h=N_{2}(h, \Phi),
\end{aligned}
$$

with the nonlinear terms defined by

$$
\begin{aligned}
& N_{1}(h, \Phi)=-\nabla \cdot(h \nabla \Phi)+\frac{1}{2} \nabla^{2}\left(h^{2} \hat{D} \Phi\right)-\hat{D}(h \hat{D} \Phi)+\hat{D}\left(h \hat{D}(h \hat{D} \Phi)+\frac{1}{2} h^{2} \nabla^{2} \Phi\right), \\
& N_{2}(h, \Phi)=\frac{1}{2}(\hat{D} \Phi)^{2}-\frac{1}{2}(\nabla \Phi)^{2}-\frac{1}{2} \Gamma_{0} \nabla \cdot\left((\nabla h)(\nabla h)^{2}\right)-(\hat{D} \Phi)\left(h \nabla^{2} \Phi+\hat{D}(h \hat{D} \Phi)\right) .
\end{aligned}
$$

Here, $h(x, t)$ and $\Phi(x, t)$ are the dimensionless surface height and velocity potential, respectively, $\nabla$ is the two-dimensional gradient operator and $\hat{D}$ is a (nonlocal) operator that multiplies each Fourier component by its wavenumber: $\hat{D} \mathrm{e}^{i k \cdot x}=|\boldsymbol{k}| \mathrm{e}^{\mathrm{i} k \cdot \boldsymbol{x}}$. The parametric forcing, which is uniform here, is characterized by $G(t)=a G_{0} \cos (t)$, while the dimensionless fluid parameters are

$$
\gamma \equiv \frac{2 v k_{i j}^{2}}{\omega}, \quad \Gamma_{0} \equiv \frac{\Gamma k_{i j}^{3}}{\rho \omega^{2}}, \quad G_{0} \equiv \frac{g k_{i}}{\omega^{2}}
$$

with $g$ the usual gravitational acceleration, $\nu$ the kinematic viscosity, $\rho$ the density and $\Gamma$ the surface tension. The applied forcing frequency $\omega$ sets the timescale while the wavenumber $k_{0}$ sets the lengthscale; this wavenumber characterizes subharmonic waves and is defined by the 


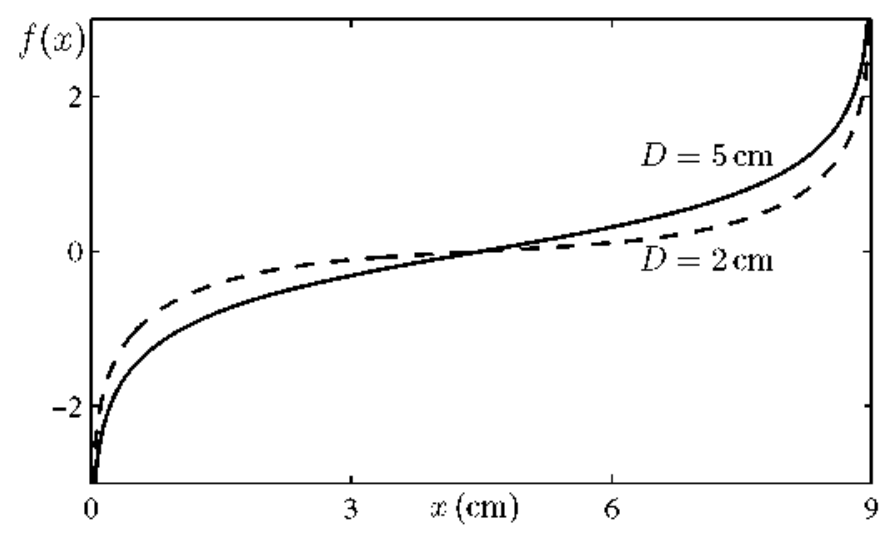

Figure 15. The shape of the induced parametric forcing described by (13) for a $9 \mathrm{~cm}$ long containet and depths of $2 \mathrm{~cm}$ (dashed curve) or $5 \mathrm{~cm}$ (solid curve).

inviscid dispersion relation

$$
g k_{0}+\frac{\Gamma k_{i}^{3}}{\rho}=\left(\frac{\omega}{2}\right)^{2}
$$

Note that (12) implies $G_{0}+\Gamma_{0}=1 / 4$.

To apply (9) to the case of horizontal forcing, the spatially uniform parametric forcing term $G(t)$ must be replaced by a nonuniform forcing term: $G(t) \rightarrow G(x, t)=$ $a G_{0} F(x) \cos (t)$. The strength of the applied horizontal acceleration is $a$ (units of $g$ ), while $F(x)$ captures the profile of the effective parametric forcing, which will be strongest near the endwalls. We take $F(x)$ from Varas and Vega (2007), where equations were derived for surface waves in horizontally vibrated containers with one extended dimension. This study identifies the parametric forcing mechanism as an 'oscillating bulk flow' generated by the motion of the endwalls. This oscillating bulk flow produces an oscillating vertical pressure gradient at the free surface that can parametrically excite subharmonic waves. The profile of this induced parametric excitation is given by

$$
f(x)=\frac{4}{\pi} \sum_{n=0}^{\infty} \frac{\sinh [(2 n+1) \pi(2 x-L) /(4 D)]}{(2 n+1) \cosh [(2 n+1) \pi L /(4 D)]}
$$

where $0 \leqslant x \leqslant L$ is the horizontal variable and $D$ is the depth of the container.

The shape of the function $f(x)$ is shown in figure 15 for $L=9 \mathrm{~cm}$ and depths of 2 and $5 \mathrm{~cm}$, corresponding to the experiment. It should be noted that there is a logarithmic singularity in $f(x)$ at $x=0, L$, where a boundary layer treatment is required and $f(x)$ does not apply. The size of this boundary layer scales as $\delta=\sqrt{v / \omega}$. Here we are not concerned with the details of the boundary layer but, consistent with the level of approximation inherent in (9), simply set the parametric forcing $F(x)$ to zero when $x \leqslant \delta$ or $L-x \leqslant \delta$. This procedure ignores both forcing and damping within these boundary layers (just as lateral boundaries are ignored). For the experimentally relevant parameters, it is sufficient to truncate the summation in (13) at 100 terms.

The appropriate numerical factor relating $F(x)$ with $f(x)$ can be obtained by comparing the amplitude equations for subharmonic waves derived in Varas and Vega (2007) 
(see (5.3) therein) with those derived from (9) in Porter and Silber (2004). This comparison yields $F(x)=\left(2 k_{0} / k_{h}\right) f(x)$, where $k_{h}$ is the wavenumber of inviscid harmonic waves satisfying

$$
g k_{h}+\frac{\Gamma k_{h}^{3}}{\rho}=\omega^{2} .
$$

We emphasize that the forcing $G(x, t)=a G_{0} F(x) \cos (t)$ substituted into $(9)$ is a natural generalization of the usual uniform parametric forcing, while its particular profile $F(x)$ is based on a theoretical result (Varas and Vega 2007). In other systems with localized parametric forcing the profile may differ from $F(x)$, but the forcing term will still take the same general form.

We integrate (9) after substituting $G(t) \rightarrow a G_{0} F(x) \cos (t)$ using the Fourier-Galerkin method outlined in Zhang and Viñals (1998). This is a convenient way to deal with the nonlocal operator $\hat{D}$, which is diagonal in Fourier space. Time stepping is done using a trapezoidal scheme for linear terms and a second-order Adams-Bashforth scheme for nonlinear terms (the parametric forcing term $G(x, t) h(x, y)$ is treated as a nonlinear term). As noted by Zhang and Viñals (1998), high wavenumber instabilities can appear after long integration times, and we have likewise found it necessary to apply a low-pass filter.

Periodic boundary conditions in $x \in[0, L]$ are not appropriate in our case because the motion of the two endwalls is out-of-phase by $180^{\circ}$ and thus the opposing subharmonic waves, to the extent that they are independent of each other near these endwalls, are out-of-phase by $90^{\circ}$. Neumann boundary conditions (vanishing perpendicular derivative at the boundaries) are more appropriate, particularly in the case of an underfilled container (i.e. 'free' boundary conditions), and are imposed in what follows.

\subsection{Onset of subhamonic waves}

The onset at $a=a_{c}$ of subharmonic waves as a function of forcing frequency is shown in figure 16. These results are obtained by integrating the linearized version of (9) with parameters appropriate for $5 \mathrm{cSt}$ silicone oil on a $9 \mathrm{~cm}$ square domain with Neumann boundary conditions. The calculated onset values are in rough qualitative agreement with the experiment (cf figure 7), but underestimate the critical acceleration by a factor of two or so over most of the range, probably due to damping within the boundary layers and at the contact line, which is neglected in the model. Note the oscillations in $a_{c}$ as the minimum onset value moves to higher modes with increasing forcing frequency. These oscillations are due to discrete wavenumber selection in the crosswise $y$-direction: $k_{y}=N \pi / L, N=1,2, \ldots$, and reflect the detuning between the selected frequency $\omega / 2$ and the natural frequencies of the allowed modes. This is illustrated more clearly in the upper part of figure 17 where the onset for individual modes is shown between 60 and $70 \mathrm{~Hz}$. The lower part of figure 17 shows the average pattern angle for the mode with the lowest onset. Not surprisingly, the (linear) detuning in a $9 \mathrm{~cm}$ square container can cause the eigenfunctions to rotate; the average pattern angle can change by as much as $10^{\circ}$ as the forcing frequency sweeps past (twice) the natural frequency of one mode and on to the next.

The first two excited modes at $64 \mathrm{~Hz}$ are shown in figure 18 . The $N=31$ mode (a) has the lowest onset, and at $64 \mathrm{~Hz}$ this is very close to the minimum of the neutral stability curve (at $63.7 \mathrm{~Hz}$ ). The next instability is to the $N=30$ mode (b), which has one less (half) oscillation in $y$ and, to compensate, more rapid oscillations in $x$. 


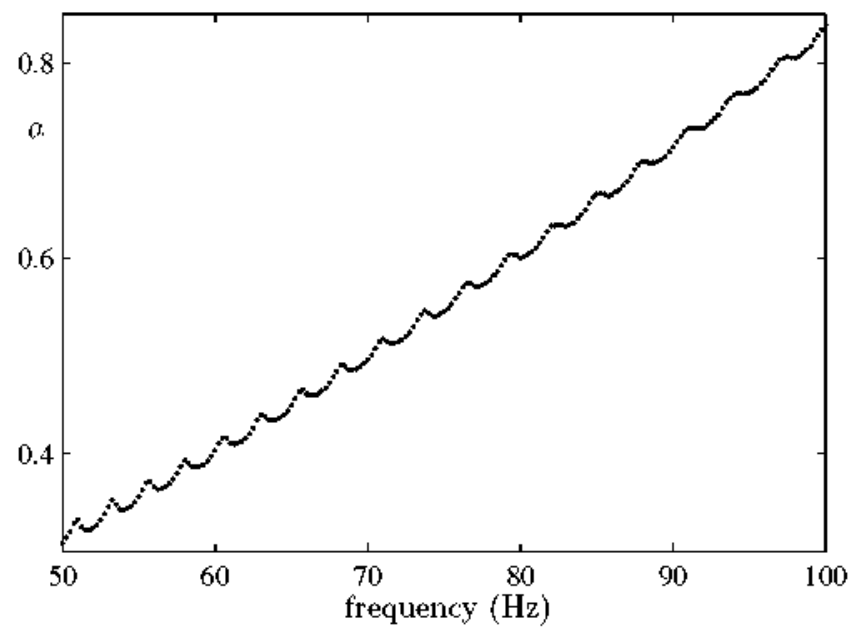

Figure 16. Onset value (in units of $g$ ) versus ftequency between 50 and $100 \mathrm{~Hz}$ in a $9 \mathrm{~cm}$ square container: Parameters correspond to a $5 \mathrm{~cm}$ deep layer of $5 \mathrm{cSt}$ silicone oil.

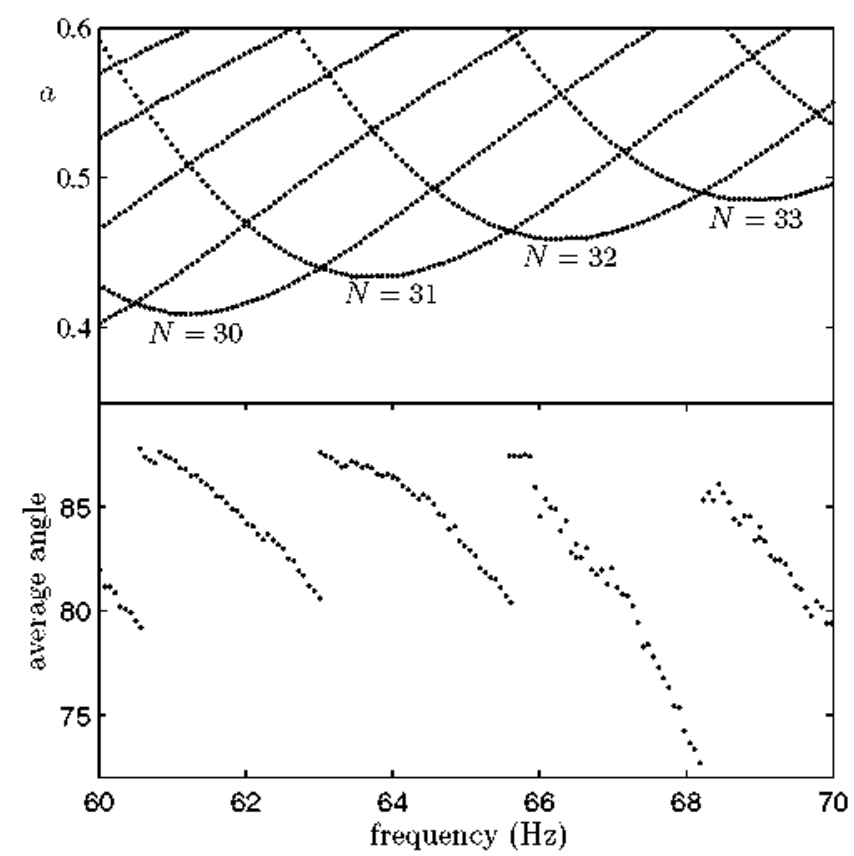

Figure 17. Upper: onset values (in units of $g$ ) for selected modes with $k_{y}=N \pi / L$ between 60 and $70 \mathrm{~Hz}$ in a $9 \mathrm{~cm}$ square container. Lower: average pattern angle for the mode with lowest onset. Parameters correspond to a $5 \mathrm{~cm}$ deep layer of $5 \mathrm{cSt}$ silicone oil.

\subsection{Bifurcation curves}

We can use (9) to make a more direct comparison with experiment by varying the forcing amplitude $a$ with frequency fixed, as in section 3 . The simulation results for $5 \mathrm{cSt}$ oil in a $5 \mathrm{~cm}$ deep container are shown in figure 19, which should be compared with figure 7 . Note that, while some bifurcations are supercritical, others are clearly subcritical (at 80 and $100 \mathrm{~Hz}$, for example). This is consistent with the experiments (supercriticality or weak subcriticality 
(a)

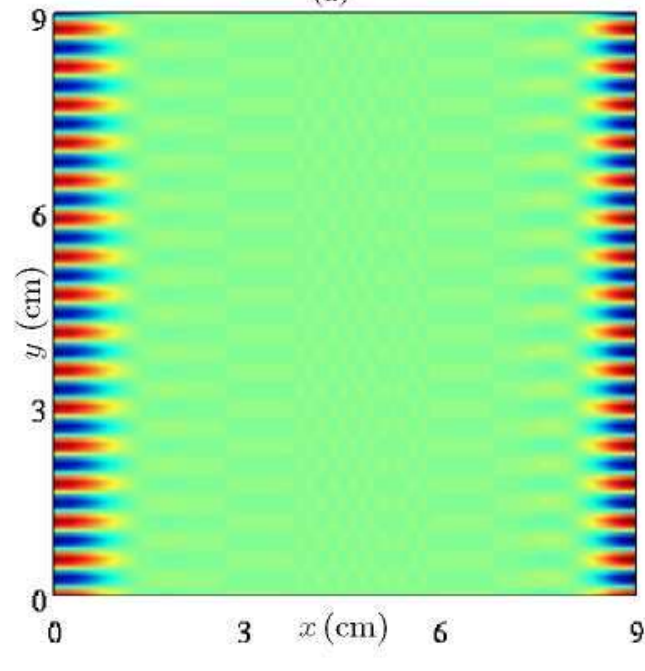

(b)

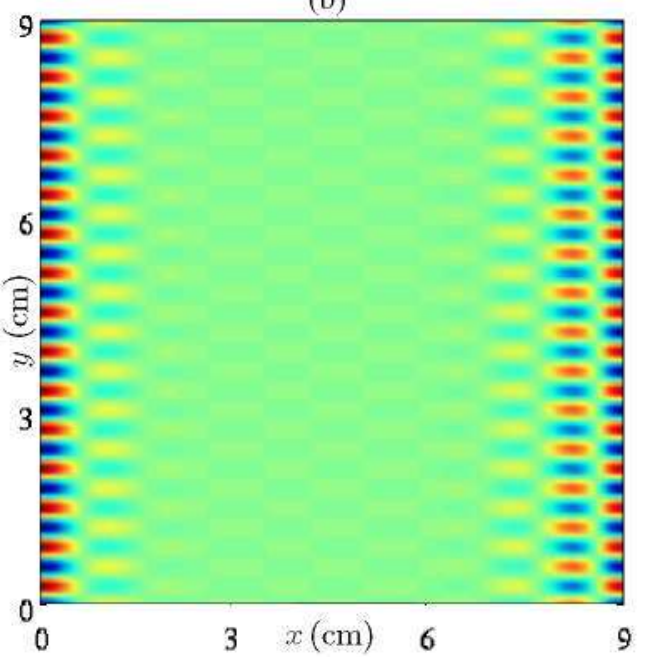

Figure 18. Patterns corresponding to the first two instabilities at $64 \mathrm{~Hz}$ with the parameters of figure 17: (a) $N=31$ and (b) $N=30$.

is observed) and with the known effect of detuning on parametric instabilities (Meron 1987, Lichter and Bernoff 1988): supercritical bifurcation is expected when the forcing frequency is above the resonance frequency, subcritical bifurcation when it is below. The lower portion of the figure shows that in the simulations, as in the experiment, the pattern angle tends to decrease as the forcing and wave amplitude increase. The difference between the onset pattern and the nonlinear pattern obtained at higher forcing is illustrated in figure 20 . To the eye, there is not much separating these solutions besides the modulations evident on the right side of figure 20(b). This suggests that, as in the experiments, the nonlinear solutions (prior to any secondary instabilities) are not qualitatively different from the eigenfunctions of the linear problem.

Simulation results for $10 \mathrm{cSt}$ oil in a $5 \mathrm{~cm}$ deep container are shown in figure 21 , which should be compared with figure 10. Again, the bifurcation of subharmonic patterns is either supercritical or (slightly) subcritical, depending on the detuning at the applied frequencies. The pattern angle decreases with increasing amplitude, but less so than in the lower viscosity case of figure 19.

The numerical results for $50 \mathrm{cSt}$ oil in a $5 \mathrm{~cm}$ deep container are shown in figure 22 , which can be compared with figure 11 . There is little to distinguish between supercritical and subcritical bifurcations now, nor is there much evidence that the pattern angle is changing with forcing amplitude. Recall, however, that (9) is not expected to work very well at such large viscosity.

\subsection{Dependence on depth}

The container depth, which sets the scale of the forcing $F(x)$, can be easily varied in the numerical model and the results of this are shown in figure 23 for the case of $60 \mathrm{~Hz}$ forcing and $5 \mathrm{cSt}$ oil. The critical forcing value decreases with increasing depth, as expected. Note that $5 \mathrm{~cm}$ is effectively in the deep layer limit; the onset is not appreciably lowered by further increasing fluid depth. 


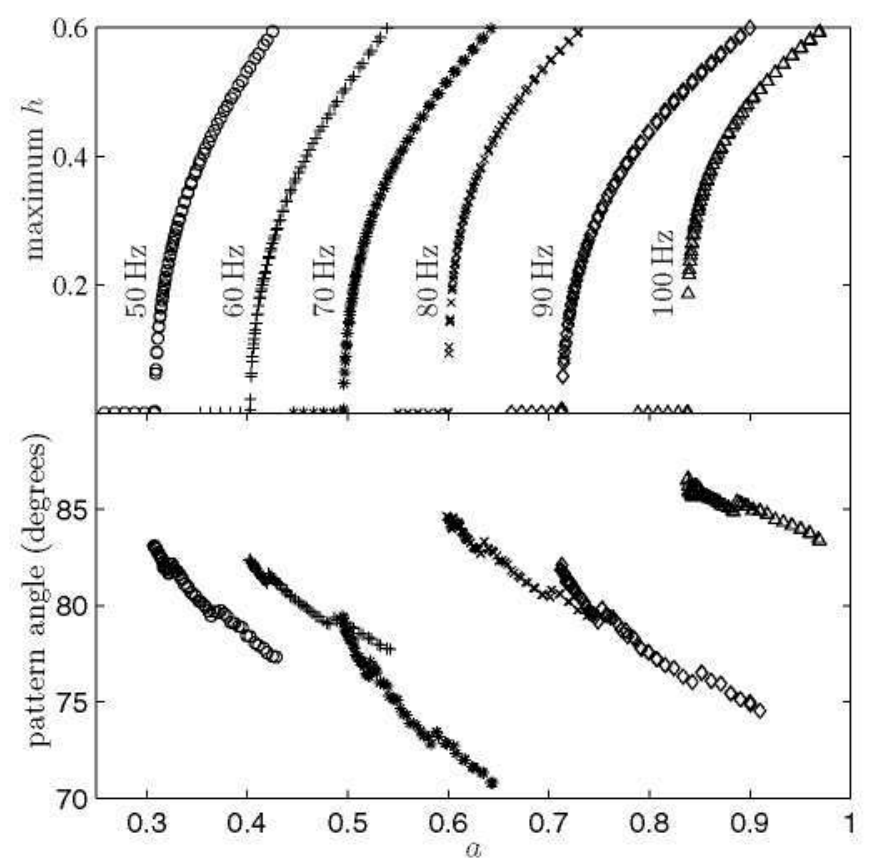

Figure 19. Upper: bifurcation of subharmonic patterns in a $9 \mathrm{~cm}$ square container with forcing of $50-100 \mathrm{~Hz}$. The surface height $h$, maximized over space and time, is shown. Lower: average pattern angle of these solutions. Parameters correspond to a $5 \mathrm{~cm}$ deep layer of $5 \mathrm{cSt}$ silicone oil.

(a)

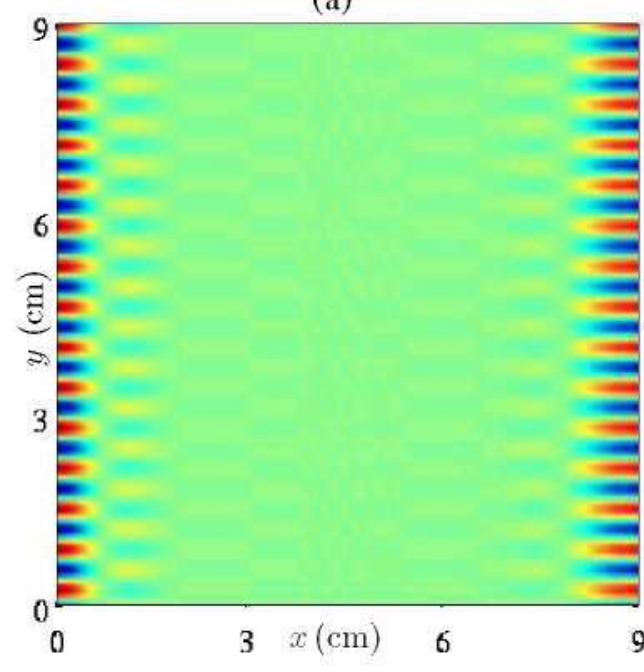

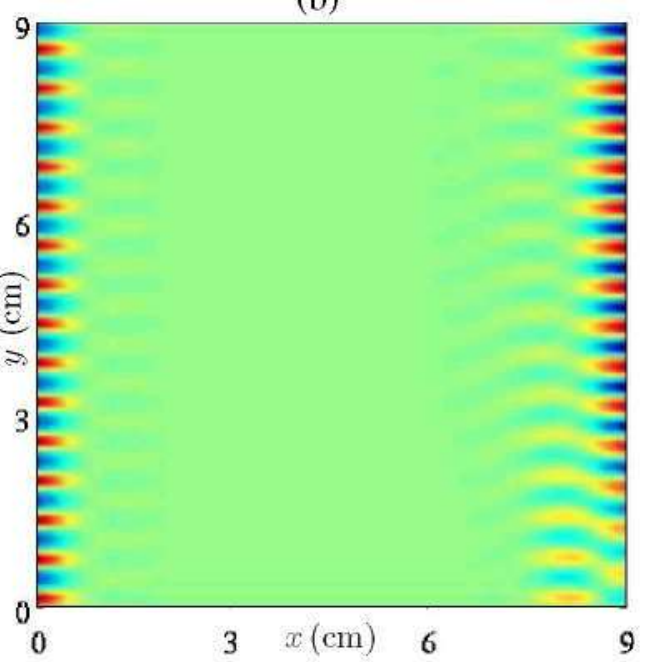

(b)

Figure 20. Patterns obtained with (9) corresponding to a $9 \mathrm{~cm}$ square container of $5 \mathrm{cSt}$ silicone oil forced at $60 \mathrm{~Hz}$. (a) Surface pattern at onset: $a=0.403$. (b) Surface pattern well above onset: $a=0.542$.

\subsection{Dependence on viscosity}

The effect of increasing viscosity in the experiment is to raise the threshold of subharmonic instability and to lower the average pattern angle. The results of continuously increasing 


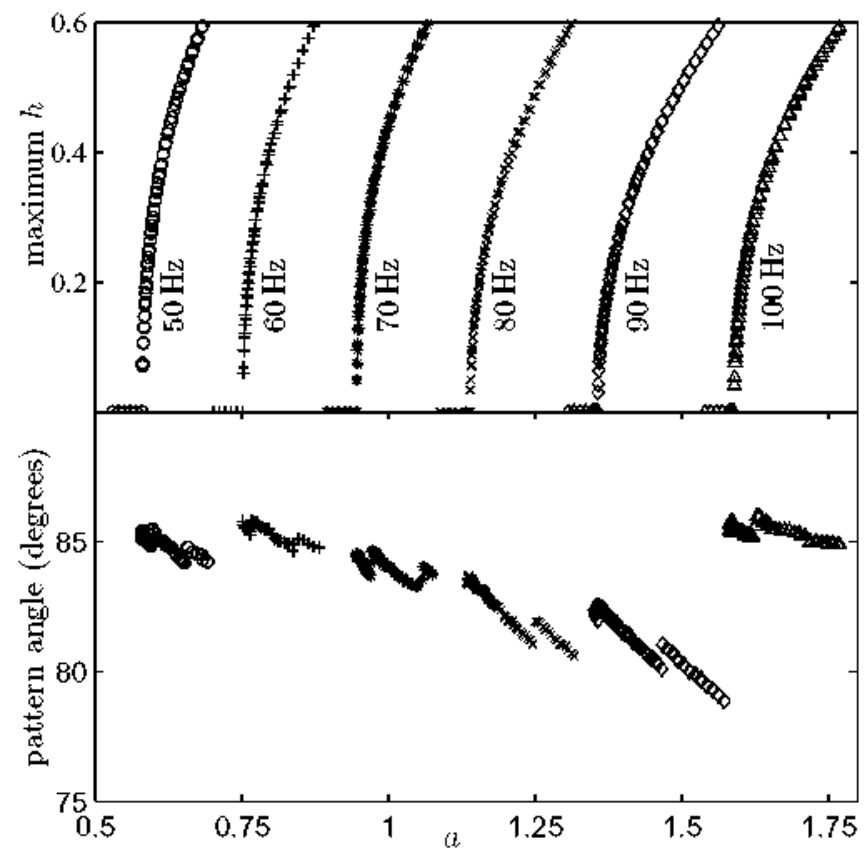

Figure 21. Upper: bifurcation of subharmonic patterns in a $9 \mathrm{~cm}$ square container with forcing of $50-100 \mathrm{~Hz}$. The surface heisht $h$, maximized over space and time, is shown. Lower: average pattern angle of these solutions. Patameters correspond to a $5 \mathrm{~cm}$ deep layer of $10 \mathrm{cSt}$ silicone oil.

viscosity in the numerical model are shown in figure 24 , again for the case of $60 \mathrm{~Hz}$ forcing. The onset of the subharmonic instability increases with viscosity $v$ in a nearly linear fashion, particularly for small values. This should be contrasted with the $\sqrt{v}$ dependence predicted by the NLS model.

The patten angle becomes increasingly difficult to measure at higher viscosities because the patterns become very localized near the endwalls and the corresponding Fourier transform is therefore very broad. The overlap of symmetric peaks at $\left(k_{x}, k_{y}\right)$ and $\left(-k_{x}, k_{y}\right)$ in this transform can cause the maximum to move to $k_{x}=0$ (corresponding to a pattern angle of $90^{\circ}$ ). This happens for an increasing fraction of the oscillation period as viscosity increases and suggests that the location of the maximum of the spectrum, averaged over time, no longer provides a good measure of highly localized patterns. The average angle remains roughly constant, but the minimum angle, which is taken from the point of the oscillation period when the peaks in the Fourier transform are most separated from each other, reveals a trend toward lower angles (more rotated patterns) as viscosity increases, consistent with the experimental results of figures 7,10 and 11 .

\subsection{Discussion of numerical results}

Here we summarize the conclusions that can be drawn from the numerical simulations of this section. First, the subharmonic patterns observed in the experiments can be captured surprisingly well by a model containing a spatially dependent parametric forcing term (as opposed to forcing via boundary condition as in the NLS model). This model correctly captures several features including the increase in streamwise modulation (decrease in pattern angle) with wave amplitude and the apparent (but weak) decrease in pattern angle with 


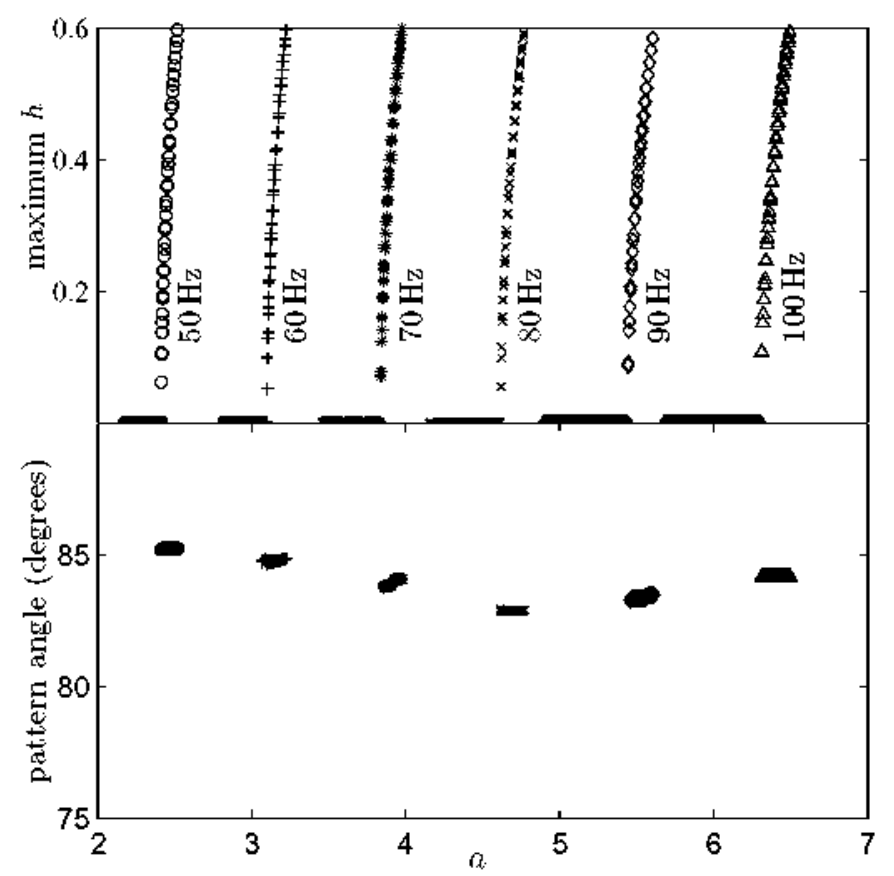

Figure 22. Upper: bifurcation of subharmonic patterns in a $9 \mathrm{~cm}$ square container with forcing of $50-100 \mathrm{~Hz}$. The surface height $h$, maximized over space and time, is shown. Lower: average pattern angle of these solutions. Parameters correspond to a $5 \mathrm{~cm}$ deep layer of $50 \mathrm{cSt}$ silicone oil.

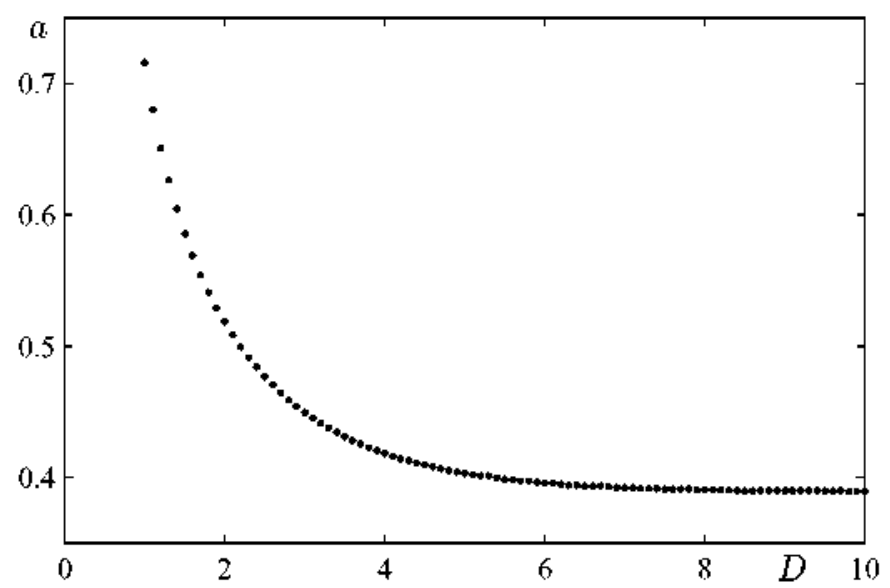

Figure 23. Onset $a$ (in units of $g$ ) versus depth $D$ (in $\mathrm{cm}$ ) in a $9 \mathrm{~cm}$ square container with $60 \mathrm{~Hz}$ forcing. Parameters correspond to $5 \mathrm{eSt}$ silicone oil.

viscosity. Second, the simplified damping and boundary conditions used in the model suggests that the patterns observed in the experiments are quite generic. Their properties may be affected by, but do not rely on, the precise boundary conditions and other experimental details. Third, these patterns seem to be largely determined by the linear problem. That is, while nonlinear effects do come into play, typically lowering the pattern angle as noted above, the qualitative character of these patterns is present already in the linear eigenfunctions. This 


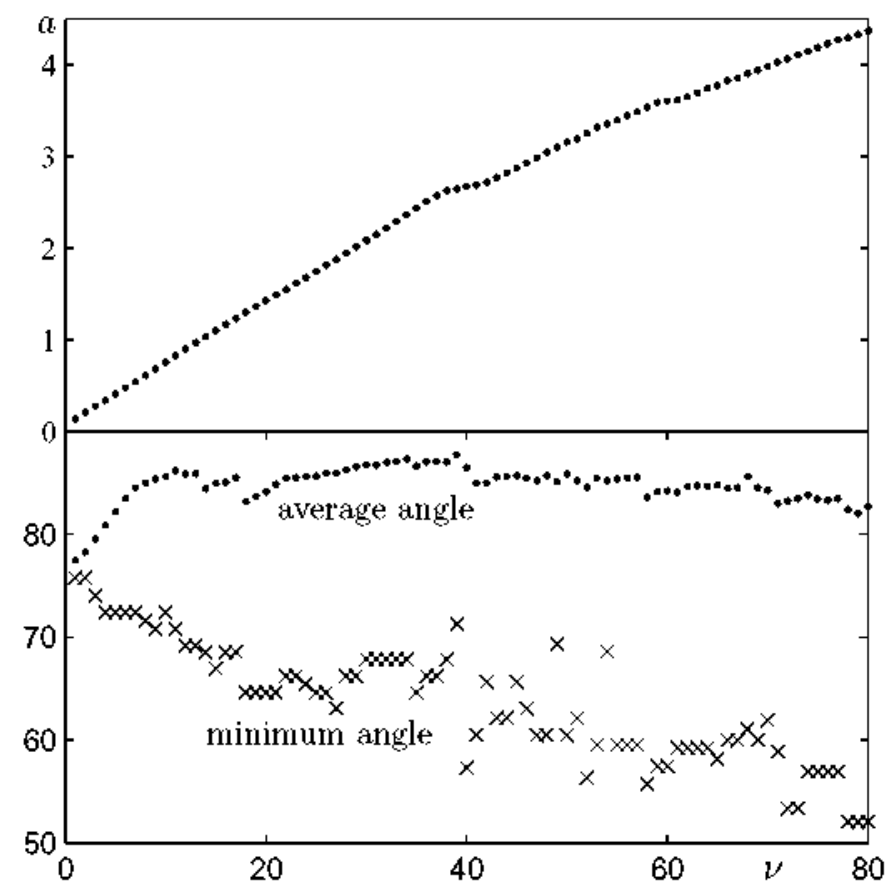

Figure 24. Upper: onset $a$ (in $g$ 's) versus viscosity $v$ (in cSt) in a $9 \mathrm{~cm}$ square container, $5 \mathrm{~cm}$ deep, with $60 \mathrm{~Hz}$ forcing. Lower: average and minimum pattern angle versus viscosity.

suggests that wavenumber selection in the streamwise direction is fundamentally due to the spatial profile of the parametric forcing mechanism and not the result of resonant interactions between the subharmonic waves themselves.

\section{Conclusion}

The cross-wave instability is richer and more closely related to the subharmonic standing wave instability of vertically forced Faraday systems than has commonly been appreciated, despite Faraday having made this point himself (Faraday 1831):

118. These ripple-like stationary undulations are perfectly analogous as to cause, arrangement and action with the heaps and crispations already explained, i.e. they are the results of that vibrating motion in directions perpendicular to the force applied (105), by which the water can most readily accommodate itself to rapid, regular, and alternating changes in bulk in the immediate neighbourhood of the oscillating parts.

This connection has been neglected due to the different treatment these waves have received in subsequent experiments. While vertically excited subharmonic waves were systematically investigated across a wide range of parameters, including large-aspect-ratio containers and moderate damping where a variety of beautiful patterns were discovered (see, e.g. Edwards and Fauve 1994, Kudrolli et al 1998, Arbell and Fineberg 2002), the interest in cross-waves has been largely confined to the context of low frequency wavemaker experiments in water. The theory (Jones 1984, Lichter and Chen 1987, Lichter and Bernoff 1988, Miles 1988, Bernoff et al 1989, Shemer and Kit 1989, Ayanle et al 1990, Shemer and Lichter 1990) 
developed to describe these cross-wave experiments focused on a NLS description that is valid in the limit of small damping and forcing, when the waves are close to ideal crosswaves, and where they vary slowly compared to the size of the forcing region (comparable to the depth of the wavemaker).

The experiments described in section 3 demonstrate the presence in horizontally vibrated systems of subharmonic surface waves that do not conform to the standard description of cross-waves and where no separation of lengthscales occurs. These waves appear at onset, persist over a wide range of parameters, and are undoubtedly the same basic phenomenon described by Faraday in 1831. The patterns we observe, however, cannot properly be called 'cross-waves'. They are characterized by an average angle typically between $50^{\circ}$ and $80^{\circ}$. This angle is difficult to measure precisely (due to the rapid spatial decay of the patterns) and can vary with forcing frequency (detuning), forcing amplitude, viscosity and boundary conditions. Under no circumstances, however, did we observe pure crosswise patterns, leading us to conclude that they simply do not arise for the range of parameters considered.

Some properties of these patterns were understood using a modified version of a Faraday wave model derived by Zhang and Viñals (1997) atter replacing the uniform parametric forcing term with an appropriate spatially dependent one. This model easily produces the same type of pattern observed in the experiments, albeit with slightly higher angles, and gives onset values that are in qualitative agreement. The detuning is seen to select between supercritical and subcritical bifurcation, as expected, and to generate variations in average pattern angle of $10^{\circ}$ or so in a $9 \mathrm{~cm}$ square container. The decrease of pattern angle with forcing amplitude, observed in figures $4,7,9,10$ and 11 , is also captured by the model (except at high viscosity). Furthermore, figure 24 supports the notion that viscosity is important in the rotation of patterns away from $90^{\circ}$. At the same time, there is no evidence that pure crosswise patterns exist in this system, even in the inviscid limit.

A physical argument to explain rotated patterns begins with the basic fact that, already at the linear level, the surface waves obey a dispersion relation that depends on forcing as well as damping. If this forcing varies with $x$, as it does in our case, then some kind of modulation in $x$ will be induced on the same scale. Near the inviscid limit, where the critical forcing is very small and the dispersion relation is nearly that of inviscid (unforced) capillarygravity waves, cross-waves that exhibit only very slow oscillations in the $x$-direction should be present, consistent with the observations of low frequency wavemaker experiments (Barnard and Pritchard 1972, Lichter and Shemer 1986, Shemer and Lichter 1987, 1990, Lichter and Underhill 1987, Shemer and Kit 1989, Underhill et al 1991). Indeed, we find some evidence for this 'cross-wave' limit both experimentally, in the more rotated patterns obtained with the more viscous $50 \mathrm{cSt}$ oil (figure 11), and numerically, in the observed correlation between viscosity and pattern angle shown in figure 24 . We emphasize again, however, that pure cross-waves were never observed in our experimental container, and not even in the numerical simulations with $v \ll 1$, probably because the forcing (with a depth of $2-5 \mathrm{~cm}$ ) is not sufficiently localized to allow the separation of lengthscales required. A more careful investigation of the inviscid limit, considering both short and long containers would be illuminating and will be undertaken elsewhere.

\section{Acknowledgments}

This work was supported by the Spanish Ministerio de Educación y Ciencia projects ESP2006-03091 and ESP2007-65221 and Ministerio de Ciencia e Innovación project AYA2010-19081. 


\section{References}

Arbell H and Fineberg J 2002 Phys. Rev. E 65036224

Ayanle H, Bernoff A J and Lichter S 1990 Physica D 43 87-104

Barnard B J S and Pritchard W G 1972 J. Fluid Mech. 55 245-55

Benjamin T B and Ursell F 1954 Proc. R. Soc. A 225 505-15

Bernoff A J, Kwok L P and Lichter S 1989 Phys. Fluids A 1 678-88

Edwards W S and Fauve S 1994 J. Fluid Mech. 278 123-48

Faraday M 1831 Phil. Trans. R. Soc. Lond. 121 299-340

Feng Z C 1997 Phys. Rev. Lett. $79415-8$

Fisher R B and Naidu D K 1996 in Image Technology: Advances in Image Processing, Multimedia and Machine Vision (Berlin: Springer) pp 385-404

Funakoshi M and Inoue S 1988 J. Fluid Mech. 192 219-47

Garrett C J R 1970 J. Fluid Mech. 41 837-49

González-Viñas W and Salán J 1994 Europhys. Lett. 26 665-70

Gresho P M and Sani R L 1970 J. Fluid Mech. 40 783-06

Hocking L M 1987 J. Fluid Mech. 179 253-66

Jones A F 1984 J. Fluid Mech. 138 53-74

Kit E and Shemer L 1989 Phys. Fluids A 1 1128-32

Kudrolli A, Pier B and Gollub J P 1998 Physica D 123 99-111

Lichter S and Bernoff A J 1988 Phys. Rev. A 37 1663-7

Lichter S and Chen J 1987 J. Fluid Mech. 183 451-65

Lichter S and Shemer L 1986 Phys. Fluids 29 3971-5

Lichter S and Underhill W B 1987 Phys. Rev. A 35 5282-4

Lin J D and Howard L N 1960 Non-linear standing waves in a rectangular tank due to forced oscillation Hydrodynamics Laboratory Technical Report 44 MIT

McGoldrick L F 1968 Faraday waves: the cross-wave resonant instability Technical Report 2 Department of Geophysical Sciences, University of Chicago

Mei C C and Liu L F 1973 J. Fluid Mech. 59 239-56

Meron E 1987 Phys. Rev. A 35 4892-5

Miles J 1988 J. Fluid Mech. 186 119-27

Miles J and Henderson D 1990 Annu. Rev. Fluid Mech. 22 143-65

Miles J W 1984 J. Fluid Mech. 149 15-31

Perlin M and Schultz W W 2000 Annu. Rev. Fluid Mech. 32 241-74

Porter J and Silber M 2004 Physica D 190 93-114

Schuler M 1933 Z. Angew. Math. Mech. 13 443-6

Settles G S 2001 Schlieren and Shadowgraph Techniques: Visualizing Phenomena in Transparent Media (Berlin: Springer)

Shemer L and Kit E 1989 J. Fluid Mech. 209 249-63

Shemer L and Lichter S 1987 Phys. Fluids $303427-33$

Shemer L and Lichter S 1990 Exp. Fluids 9 148-52

Skeldon A C and Porter J 2011 Phys. Rev. E 84016209

Talib E, Jalikop S V and Juel A 2007 J. Fluid Mech. $\mathbf{5 8 4} 45-68$

Taneda S 1994 Fluid Dyn. Res. 14 119-51

Taneda S 1995 Fluid Dyn. Res. 16 61-70

Underhill W B, Lichter S and Bernoff A J 1991 J. Fluid Mech, 225 371-94

Varas F and Vega J M 2007 J. Fluid Mech. 579 271-304

Vega J M, Knobloch E and Martel C 2001 Physica D 154 313-36

Wolf G H 1969 Z. Phys. 227 291-300

Wolf G H 1970 Phys. Rev. Lett. 24 444-6

Wunenburger R, Evesque P, Chabot C, Garrabos Y, Fauve S and Beysens D 1999 Phys. Rev. E 59 5440-5

Zhang W and Viñals J 1997 J. Fluid Mech. 336 301-30

Zhang W and Viñals J 1998 Physica D 116 225-43 